\title{
Phylogenetic analysis demonstrating four new species in Megasporoporia sensu lato (Polyporales, Basidiomycota)
}

\author{
Wang $Y R^{1,2}$, Wu $Y^{1,3}$, Vlasák $J^{4}$, Yuan $Y^{1^{*}}$ and Dai $Y_{C}{ }^{1^{*}}$
}

${ }^{1}$ Institute of Microbiology, School of Ecology and Nature Conservation, Beijing Forestry University, Beijing 100083, China

${ }^{2}$ The college of Forestry, Beijing Forestry University, Beijing 100083, China

${ }^{3}$ China Fire and Rescue Institute, Beijing 102202, China

${ }^{4}$ Biology Centre, Czech Academy of Sciences, Institute of Plant Molecular Biology, Branišovská 31, CZ-370 05 České Budějovice, Czech Republic

Wang YR, Wu YD, Vlasák J, Yuan Y, Dai YC 2021 - Phylogenetic analysis demonstrating four new species in Megasporoporia sensu lato (Polyporales, Basidiomycota). Mycosphere 12(1), 1012-1037, Doi 10.5943/mycosphere/12/1/11

\begin{abstract}
Megasporoporia sensu lato is a common polypore genus in tropics, the members of which are readily recognized in the field because of their resupinate, light-coloured basidiocarps with big pores. The species of the genus usually grow on fresh fallen trunks and branches. The genus was recognized as polyphyletic by molecular analyses and divided into three genera: Megasporia, Megasporoporia sensu stricto and Megasporoporiella. In the present study, phylogenies based on the combined 2-gene (ITS + nLSU) and 4-gene (ITS + nLSU + mtSSU + TEF) sequences datasets of Megasporoporia sensu lato are analysed, and 21 lineages nested in three clades (the Megasporia clade, the Megasporoporia sensu stricto clade and the Megasporoporiella clade) are formed. Based on morphological examination, four new species, Megasporia bambusae, Megasporia fusiformis, Megasporoporia inflata and Megasporoporiella australiae, are described based on materials from Australia, China and Malaysia, and a new combination, Megasporoporiella hubeiensis comb. nov., is proposed. Megasporoporiella pseudocavernulosa is selected as the type species of Megasporoporiella because the previous type species Megasporoporiella cavernulosa was misidentified. Polyporus megasporoporus is the revised name for Megasporoporiella lacerata. In addition, a comparison of main characteristics and an identification key of Megasporoporia sensu lato are provided.
\end{abstract}

Keywords - morphology - phylogeny - polymerase - Polyporaceae - taxonomy

\section{Introduction}

Ryvarden et al. (1982) established Megasporoporia Ryvarden \& J.E. Wright in 1982, and four species were addressed in the genus. Species of the genus are easily recognized in the field because of their light coloured, resupinate basidiocarps with big pores. Microscopically, the genus is characterized by a dimitic hyphal structure with generative hyphae bearing clamp connections, skeletal hyphae usually branched and dextrinoid, presence of cystidioles and dendrohyphidia in most species, and hyaline, thin-walled, big basidiospores (Ryvarden et al. 1982, Dai et al. 2004, Li $\&$ Cui 2013a, Yuan et al. 2017, Cui et al. 2019). Ecologically, the major members of the genus have a distribution in tropics, and usually grow on fallen trunks, branches and twigs which are not 
much decayed. The species diversity was for long underestimated, and 14 species were described recently (Dai \& Li 2002, Dai \& Wu 2004, Cui \& Dai 2007, Zhou \& Dai 2008, Du \& Cui 2009, Li \& Cui 2013a, Yuan et al. 2017). In addition, the molecular phylogeny demonstrated that the genus is polyphyletic, and two genera - Megasporia B.K. Cui et al. and Megasporoporiella B.K. Cui et al. were derived from Megasporoporia (Li \& Cui 2013a). So, the definition of Megasporoporia by Ryvarden et al. (1982) is in sensu lato, including three genera: Megasporia, Megasporoporia sensu stricto and Megasporoporiella (Li \& Cui 2013a). These three genera are distinctly different in phylogeny, but it is very difficult to distinguish them morphologically because their major characteristics are overlapped.

Based on more samples of Megasporoporia sensu lato from Australia, China and Malaysia, and using combined 2-gene (ITS + nLSU) and 4-gene (ITS + nLSU + mtSSU + TEF) sequences datasets, further phylogenetic analyses on the genus are carried out. Four new species belonging to Megasporia, Megasporoporia sensu stricto and Megasporoporiella are detected, and their illustrated descriptions are provided. Dichomitus hubeiensis Hai J. Li \& B.K. Cui is nested in the Megasporoporiella clade, and its morphology fits well Megasporoporiella, so the combination of Megasporoporiella hubeiensis is proposed.

\section{Materials \& Methods}

\section{Morphological studies}

The studied specimens are deposited in the herbaria of the Institute of Microbiology, Beijing Forestry University (BJFC), the Institute of Applied Ecology, Chinese Academy of Sciences (IFP), Universidade Federal de Pernambuco (URM), and the private herbarium of Josef Vlasák (JV). Morphological descriptions are based on field notes and herbarium specimens. Microscopic analyses follow $\mathrm{Li} \& \mathrm{Cui}$ (2013a). In the description: $\mathrm{CB}=$ Cotton Blue, $\mathrm{CB}+=$ cyanophilous in Cotton Blue, $\mathrm{CB}-=$ acyanophilous in Cotton Blue, IKI $=$ Melzer's reagent, IKI $-=$ neither amyloid nor dextrinoid, $\mathrm{KOH}=2 \%$ potassium hydroxide, $\mathrm{L}=$ arithmetic average of all spore length, $\mathrm{W}=$ arithmetic average of all spore width, $\mathrm{Q}=\mathrm{L} / \mathrm{W}$ ratios, and $\mathrm{n}=$ number of spores/measured from given number of specimens (Yuan et al. 2017). Color terms are cited from Anonymous (1969) and Petersen (1996).

\section{DNA extraction, PCR amplification and sequencing}

The total genomic DNA was extracted from the dried specimens using CTAB rapid plant genome extraction kit (Aidlab Biotechnologies Co., Ltd, Beijing), according to the manufacturer's instructions with some modifications (Chen et al. 2016, Shen et al. 2019). The PCR primers for all genes are listed in Table 1. The PCR protocol for ITS, nLSU, mtSSU, and TEF were followed by Rehner \& Buckley (2005) and Li \& Cui (2013a), and some adjustments were made to suit different species of Megasporoporia sensu lato. The PCR products were purified and sequenced in Beijing Genomics Institute (China) with the same primers. All newly generated sequences were deposited at GenBank (http://www.ncbi.nlm.nih.gov/) and listed in Table 2.

Table 1 PCR primers used in this study

\begin{tabular}{llll}
\hline Gene & Primer & ${\text { Primer sequences }\left(\mathbf{5}^{\prime} \mathbf{- 3}^{\prime}\right)^{\mathbf{a}}}{ }^{\text {a }}$ & Reference \\
\hline ITS & ITS5 & GGA AGT AAA AGT CGT AAC AAG G & White et al. 1990 \\
& ITS4 & TCC TCC GCT TAT TGA TAT GC & White et al. 1990 \\
nLSU & LR0R & ACC CGC TGA ACT TAA GC & Vilgalys \& Hester 1990 \\
& LR7 & TAC TAC CAC CAA GAT CT & Vilgalys \& Hester 1990 \\
mtSSU & MS1 & CAG CAG TCA AGA ATA TTA GTC AAT G & White et al. 1990 \\
& MS2 & GCG GAT TAT CGA ATT AAA TAA C & White et al. 1990 \\
TEF & 983F & GCY CCY GGH CAY CGT GAY TTY AT & Rehner \& Buckley 2005 \\
& 1567R & ACH GTR CCR ATA CCA CCR ATC TT & Rehner \& Buckley 2005 \\
\hline
\end{tabular}


${ }^{\text {a }}$ Degeneracr codes: $\mathrm{S}=\mathrm{G}$ or $\mathrm{C}, \mathrm{W}=\mathrm{A}$ or $\mathrm{T}, \mathrm{R}=\mathrm{A}$ or $\mathrm{G}, \mathrm{Y}=\mathrm{C}$ or $\mathrm{T}, \mathrm{N}=\mathrm{A}$ or $\mathrm{T}$ or $\mathrm{C}$ or $\mathrm{G}, \mathrm{D}=\mathrm{G}$ or $\mathrm{A}$ or $\mathrm{T}, \mathrm{M}=\mathrm{A}$ or $\mathrm{C}$.

Table 2 Information on samples of Megasporoporia sensu lato used in this study

\begin{tabular}{|c|c|c|c|c|c|c|c|}
\hline \multirow[t]{2}{*}{ Species } & \multirow[t]{2}{*}{ Sample no. } & \multirow{2}{*}{$\begin{array}{l}\text { Geographic } \\
\text { origin }\end{array}$} & \multicolumn{4}{|c|}{ GenBank accessions } & \multirow[t]{2}{*}{ References } \\
\hline & & & ITS & nLSU & mtSSU & TEF & \\
\hline Cerioporus squamosus & Cui10394 & China & $\begin{array}{l}\text { KX8516 } \\
35\end{array}$ & $\begin{array}{l}\text { KX8516 } \\
88\end{array}$ & $\begin{array}{l}\text { KX8517 } \\
14\end{array}$ & $\begin{array}{l}\text { KX8517 } \\
89\end{array}$ & $\begin{array}{l}\text { Cui et al. } \\
2019\end{array}$ \\
\hline C. squamosus & Cui10595 & China & $\begin{array}{l}\text { KU1897 } \\
78\end{array}$ & $\begin{array}{l}\text { KU1898 } \\
09\end{array}$ & $\begin{array}{l}\text { KU1899 } \\
60\end{array}$ & $\begin{array}{l}\text { KU1899 } \\
25\end{array}$ & $\begin{array}{l}\text { Zhou et al. } \\
2016\end{array}$ \\
\hline $\begin{array}{l}\text { Crassisporus } \\
\text { macroporus }\end{array}$ & Cui14468 & China & $\begin{array}{l}\text { MK1164 } \\
86\end{array}$ & $\begin{array}{l}\text { MK1164 } \\
95\end{array}$ & $\begin{array}{l}\text { MK1165 } \\
05\end{array}$ & $\begin{array}{l}\text { MK1229 } \\
84\end{array}$ & $\begin{array}{l}\text { Cui et al. } \\
2019\end{array}$ \\
\hline C. macroporus & Cui14465 & China & $\begin{array}{l}\text { MK1164 } \\
85\end{array}$ & $\begin{array}{l}\text { MK1164 } \\
94\end{array}$ & $\begin{array}{l}\text { MK1165 } \\
04\end{array}$ & $\begin{array}{l}\text { MK1229 } \\
83\end{array}$ & $\begin{array}{l}\text { Cui et al. } \\
2019\end{array}$ \\
\hline C. imbricatus & Dai 10788 & China & $\begin{array}{l}\mathrm{KC} 8673 \\
50\end{array}$ & $\begin{array}{l}\mathrm{KC} 8674 \\
25\end{array}$ & $\begin{array}{l}\text { KX8383 } \\
74\end{array}$ & - & $\begin{array}{l}\text { Cui et al. } \\
2019\end{array}$ \\
\hline C. imbricatus & Cui6556 & China & $\begin{array}{l}\text { KC8673 } \\
51\end{array}$ & $\begin{array}{l}\text { KC } 8674 \\
26\end{array}$ & - & - & $\begin{array}{l}\text { Cui et al. } \\
2019\end{array}$ \\
\hline $\begin{array}{l}\text { Daedaleopsis } \\
\text { confragosa }\end{array}$ & Cui6892 & China & $\begin{array}{l}\text { KU } 8924 \\
28\end{array}$ & $\begin{array}{l}\text { KU } 8924 \\
48\end{array}$ & $\begin{array}{l}\text { KX8383 } \\
81\end{array}$ & $\begin{array}{l}\text { KX8384 } \\
18\end{array}$ & $\begin{array}{l}\text { Cui et al. } \\
2019\end{array}$ \\
\hline D. confragosa & Cui9756 & China & $\begin{array}{l}\text { KU8924 } \\
38\end{array}$ & $\begin{array}{l}\text { KU8924 } \\
51\end{array}$ & - & - & $\begin{array}{l}\text { Cui et al. } \\
2019\end{array}$ \\
\hline D. hainanensis & Dai9268 & China & $\begin{array}{l}\text { KU8924 } \\
34\end{array}$ & $\begin{array}{l}\text { KU } 8924 \\
58\end{array}$ & $\begin{array}{l}\text { KX8384 } \\
14\end{array}$ & - & $\begin{array}{l}\text { Li et al. } \\
2016\end{array}$ \\
\hline D. hainanensis & Cui5178 & China & $\begin{array}{l}\text { KU8924 } \\
35\end{array}$ & $\begin{array}{l}\text { KU8924 } \\
62\end{array}$ & $\begin{array}{l}\text { KX8384 } \\
13\end{array}$ & $\begin{array}{l}\text { KX8384 } \\
41\end{array}$ & $\begin{array}{l}\text { Li et al. } \\
2016\end{array}$ \\
\hline D. purpurea & Dai8060 & Japan & $\begin{array}{l}\text { KU8924 } \\
42\end{array}$ & $\begin{array}{l}\text { KU8924 } \\
75\end{array}$ & $\begin{array}{l}\text { KX8384 } \\
09\end{array}$ & $\begin{array}{l}\text { KX8384 } \\
38\end{array}$ & $\begin{array}{l}\text { Li et al. } \\
2016\end{array}$ \\
\hline D. purpurea & Dai13583a & China & $\begin{array}{l}\text { KX8320 } \\
54\end{array}$ & $\begin{array}{l}\text { KX8320 } \\
63\end{array}$ & $\begin{array}{l}\text { KX8384 } \\
12\end{array}$ & $\begin{array}{l}\text { KX8384 } \\
40\end{array}$ & $\begin{array}{l}\text { Cui et al. } \\
2019\end{array}$ \\
\hline Datronia mollis & Dai11456 & China & $\begin{array}{l}\text { JX55925 } \\
3\end{array}$ & $\begin{array}{l}\text { JX55929 } \\
2\end{array}$ & $\begin{array}{l}\text { KX8383 } \\
88\end{array}$ & $\begin{array}{l}\text { KX8384 } \\
24\end{array}$ & $\begin{array}{l}\text { Li et al. } \\
2014\end{array}$ \\
\hline D. mollis & Dai11253 & China & $\begin{array}{l}\text { JX55925 } \\
8\end{array}$ & $\begin{array}{l}\text { JX55928 } \\
9\end{array}$ & $\begin{array}{l}\text { KX8383 } \\
87\end{array}$ & - & $\begin{array}{l}\text { Li et al. } \\
2014\end{array}$ \\
\hline D. subtropicus & Dai12883 & China & $\begin{array}{l}\text { KC4151 } \\
84\end{array}$ & $\begin{array}{l}\text { KC4151 } \\
91\end{array}$ & $\begin{array}{l}\text { KX8383 } \\
90\end{array}$ & $\begin{array}{l}\text { KX8384 } \\
27\end{array}$ & $\begin{array}{l}\text { Li et al. } \\
2014\end{array}$ \\
\hline D. subtropica & Dai12885 & China & $\begin{array}{l}\mathrm{KC} 4151 \\
85\end{array}$ & $\begin{array}{l}\text { KC4151 } \\
92\end{array}$ & $\begin{array}{l}\text { KX8383 } \\
91\end{array}$ & $\begin{array}{l}\text { KX8384 } \\
28\end{array}$ & $\begin{array}{l}\text { Li et al. } \\
2014\end{array}$ \\
\hline Dichomitus amazonicus & URM87859 & Brazil & $\begin{array}{l}\text { MW989 } \\
394\end{array}$ & $\begin{array}{l}\text { MW965 } \\
595\end{array}$ & - & - & $\begin{array}{l}\text { Present } \\
\text { study }\end{array}$ \\
\hline D. cylindrosporus & $\begin{array}{l}\text { Ryvarden } 4518 \\
6\end{array}$ & Belize & - & $\begin{array}{l}\text { JQ78043 } \\
9\end{array}$ & - & - & \\
\hline D. squalens & Cui9639 & China & $\begin{array}{l}\text { JQ78040 } \\
7\end{array}$ & $\begin{array}{l}\text { JQ78042 } \\
6\end{array}$ & $\begin{array}{l}\text { KX8384 } \\
04\end{array}$ & $\begin{array}{l}\text { KX8384 } \\
36\end{array}$ & $\begin{array}{l}\text { Li \& Cui } \\
\text { 2013a }\end{array}$ \\
\hline D. squalens & Cui9725 & China & $\begin{array}{l}\text { JQ78040 } \\
8\end{array}$ & $\begin{array}{l}\text { JQ78042 } \\
7\end{array}$ & $\begin{array}{l}\text { KX8384 } \\
03\end{array}$ & $\begin{array}{l}\text { KX8384 } \\
35\end{array}$ & $\begin{array}{l}\text { Li \& Cui } \\
\text { 2013a }\end{array}$ \\
\hline Echinochaete russiceps & Dai13868 & China & $\begin{array}{l}\text { KX8320 } \\
51\end{array}$ & $\begin{array}{l}\text { KX8320 } \\
60\end{array}$ & $\begin{array}{l}\text { KX8384 } \\
06\end{array}$ & $\begin{array}{l}\text { KX8384 } \\
37\end{array}$ & $\begin{array}{l}\text { Cui et al. } \\
2019\end{array}$ \\
\hline E. russiceps & Dai13866 & China & $\begin{array}{l}\text { KX8320 } \\
50\end{array}$ & $\begin{array}{l}\text { KX8320 } \\
59\end{array}$ & $\begin{array}{l}\text { KX8384 } \\
05\end{array}$ & - & $\begin{array}{l}\text { Cui et al. } \\
2019\end{array}$ \\
\hline Favolus acervatus & Cui11053 & China & $\begin{array}{l}\text { KU1897 } \\
74\end{array}$ & $\begin{array}{l}\text { KU1898 } \\
05\end{array}$ & $\begin{array}{l}\text { KU1899 } \\
56\end{array}$ & $\begin{array}{l}\text { KU1899 } \\
20\end{array}$ & $\begin{array}{l}\text { Zhou \& Cui } \\
2017\end{array}$ \\
\hline F. acervatus & Dai10749b & China & $\begin{array}{l}\text { KX5489 } \\
53\end{array}$ & $\begin{array}{l}\text { KX5489 } \\
79\end{array}$ & $\begin{array}{l}\text { KX5490 } \\
18\end{array}$ & $\begin{array}{l}\text { KX5490 } \\
43\end{array}$ & $\begin{array}{l}\text { Zhou \& Cui } \\
2017\end{array}$ \\
\hline$F$. niveus & Cui11129 & China & $\begin{array}{l}\text { KX5489 } \\
55\end{array}$ & $\begin{array}{l}\text { KX5489 } \\
81\end{array}$ & $\begin{array}{l}\text { KX5490 } \\
19\end{array}$ & $\begin{array}{l}\text { KX5490 } \\
45\end{array}$ & $\begin{array}{l}\text { Zhou \& Cui } \\
2017\end{array}$ \\
\hline$F$. niveus & Dai13276 & China & $\begin{array}{l}\text { KX5489 } \\
56\end{array}$ & $\begin{array}{l}\text { KX5489 } \\
82\end{array}$ & $\begin{array}{l}\text { KX5490 } \\
20\end{array}$ & $\begin{array}{l}\text { KX5490 } \\
46\end{array}$ & $\begin{array}{l}\text { Zhou \& Cui } \\
2017\end{array}$ \\
\hline
\end{tabular}


Table 2 Continued.

\begin{tabular}{|c|c|c|c|c|c|c|c|}
\hline \multirow[t]{2}{*}{ Species } & \multirow[t]{2}{*}{ Sample no. } & \multirow{2}{*}{$\begin{array}{l}\text { Geographic } \\
\text { origin }\end{array}$} & \multicolumn{4}{|c|}{ GenBank accessions } & \multirow[t]{2}{*}{ References } \\
\hline & & & ITS & nLSU & mtSSU & TEF & \\
\hline F.pseudoemerici & Cui11079 & China & $\begin{array}{l}\text { KX5489 } \\
58\end{array}$ & $\begin{array}{l}\text { KX5489 } \\
84\end{array}$ & $\begin{array}{l}\text { KX5490 } \\
22\end{array}$ & $\begin{array}{l}\text { KX5490 } \\
48\end{array}$ & $\begin{array}{l}\text { Zhou \& Cui } \\
2017\end{array}$ \\
\hline F. pseudoemerici & Cui13757 & China & $\begin{array}{l}\text { KX5489 } \\
59\end{array}$ & $\begin{array}{l}\text { KX5489 } \\
85\end{array}$ & $\begin{array}{l}\text { KX5490 } \\
23\end{array}$ & $\begin{array}{l}\text { KX5490 } \\
49\end{array}$ & $\begin{array}{l}\text { Zhou \& Cui } \\
2017\end{array}$ \\
\hline H. glabra & Dai12993 & China & $\begin{array}{l}\text { KX9006 } \\
37\end{array}$ & $\begin{array}{l}\text { KX9006 } \\
83\end{array}$ & $\begin{array}{l}\text { KX9007 } \\
33\end{array}$ & $\begin{array}{l}\text { KX9008 } \\
23\end{array}$ & $\begin{array}{l}\text { Cui et al. } \\
2019\end{array}$ \\
\hline H. glabra & Cui11367 & China & $\begin{array}{l}\text { KX9006 } \\
38\end{array}$ & $\begin{array}{l}\text { KX9006 } \\
84\end{array}$ & $\begin{array}{l}\text { KX9007 } \\
34\end{array}$ & $\begin{array}{l}\text { KX9008 } \\
24\end{array}$ & $\begin{array}{l}\text { Cui et al. } \\
2019\end{array}$ \\
\hline $\begin{array}{l}\text { Hornodermoporus } \\
\text { latissimus }\end{array}$ & Cui6625 & China & $\begin{array}{l}\text { HQ8766 } \\
04\end{array}$ & $\begin{array}{l}\text { JF70634 } \\
0\end{array}$ & $\begin{array}{l}\text { KF0510 } \\
40\end{array}$ & $\begin{array}{l}\text { KF1811 } \\
34\end{array}$ & $\begin{array}{l}\text { Zhao \& Cui } \\
2012\end{array}$ \\
\hline H. latissimus & Dai12054 & China & $\begin{array}{l}\text { KX9006 } \\
39\end{array}$ & $\begin{array}{l}\text { KX9006 } \\
86\end{array}$ & $\begin{array}{l}\text { KF2182 } \\
97\end{array}$ & $\begin{array}{l}\text { KF2863 } \\
03\end{array}$ & $\begin{array}{l}\text { Cui et al. } \\
2019\end{array}$ \\
\hline Megasporia bambusae & $\begin{array}{l}\text { Dai22106 } \\
\text { (Holotype) }\end{array}$ & China & $\begin{array}{l}\text { MW694 } \\
\mathbf{8 8 4}\end{array}$ & - & $\begin{array}{l}\text { MW694 } \\
912\end{array}$ & $\begin{array}{l}\text { MZ6186 } \\
31\end{array}$ & $\begin{array}{l}\text { Present } \\
\text { study }\end{array}$ \\
\hline M. bambusae & Dai20064 & China & $\begin{array}{l}\text { MW694 } \\
885\end{array}$ & $\begin{array}{l}\text { MW694 } \\
928\end{array}$ & $\begin{array}{l}\text { MW694 } \\
913\end{array}$ & $\begin{array}{l}\text { MZ6186 } \\
32\end{array}$ & $\begin{array}{l}\text { Present } \\
\text { study }\end{array}$ \\
\hline M. cystidiolophora & Cui2642 & China & $\begin{array}{l}\text { JQ78039 } \\
0\end{array}$ & $\begin{array}{l}\text { JQ78043 } \\
2\end{array}$ & - & - & $\begin{array}{l}\text { Li \& Cui } \\
\text { 2013a }\end{array}$ \\
\hline M. cystidiolophora & $\begin{array}{l}\text { Cui2688 } \\
\text { (Paratype) }\end{array}$ & China & $\begin{array}{l}\text { JQ78038 } \\
9\end{array}$ & $\begin{array}{l}\text { JQ78043 } \\
1\end{array}$ & - & - & $\begin{array}{l}\text { Li \& Cui } \\
2013 a\end{array}$ \\
\hline M. ellipsoidea & Dai19743 & China & $\begin{array}{l}\text { MW694 } \\
879\end{array}$ & $\begin{array}{l}\text { MW694 } \\
923\end{array}$ & $\begin{array}{l}\text { MW694 } \\
899\end{array}$ & $\begin{array}{l}\text { MZ6692 } \\
20\end{array}$ & $\begin{array}{l}\text { Present } \\
\text { study }\end{array}$ \\
\hline M. ellipsoidea & $\begin{array}{l}\text { Cui5222 } \\
\text { (Holotype) }\end{array}$ & China & $\begin{array}{l}\text { JQ31436 } \\
7\end{array}$ & $\begin{array}{l}\text { JQ31439 } \\
0\end{array}$ & - & - & $\begin{array}{l}\text { Li \& Cui } \\
\text { 2013a }\end{array}$ \\
\hline M. fusiformis & $\begin{array}{l}\text { Dai18596 } \\
\text { (Holotype) }\end{array}$ & Malaysia & $\begin{array}{l}\text { MW694 } \\
892\end{array}$ & $\begin{array}{l}\text { MW694 } \\
935\end{array}$ & $\begin{array}{l}\text { MW694 } \\
920\end{array}$ & $\begin{array}{l}\text { MZ6186 } \\
37\end{array}$ & $\begin{array}{l}\text { Present } \\
\text { study }\end{array}$ \\
\hline M. fusiformis & Dai18578 & Malaysia & $\begin{array}{l}\text { MW694 } \\
893\end{array}$ & $\begin{array}{l}\text { MW694 } \\
936\end{array}$ & $\begin{array}{l}\text { MW694 } \\
921\end{array}$ & $\begin{array}{l}\text { MZ6186 } \\
38\end{array}$ & $\begin{array}{l}\text { Present } \\
\text { study }\end{array}$ \\
\hline M. guangdongensis & $\begin{array}{l}\text { Cui9130 } \\
\text { (Holotype) }\end{array}$ & China & $\begin{array}{l}\text { JQ31437 } \\
3\end{array}$ & $\begin{array}{l}\text { JQ78042 } \\
8\end{array}$ & - & - & $\begin{array}{l}\text { Li \& Cui } \\
\text { 2013a }\end{array}$ \\
\hline M. guangdongensis & Cui13986 & China & $\begin{array}{l}\text { MG8472 } \\
08\end{array}$ & $\begin{array}{l}\text { MG8472 } \\
17\end{array}$ & $\begin{array}{l}\text { MG8472 } \\
29\end{array}$ & $\begin{array}{l}\text { MG8676 } \\
99\end{array}$ & $\begin{array}{l}\text { Cui et al. } \\
2019\end{array}$ \\
\hline M. hengduanensis & $\begin{array}{l}\text { Cui8076 } \\
\text { (Holotype) }\end{array}$ & China & $\begin{array}{l}\text { JQ78039 } \\
2\end{array}$ & $\begin{array}{l}\text { JQ78043 } \\
3\end{array}$ & $\begin{array}{l}\text { MG8472 } \\
52\end{array}$ & $\begin{array}{l}\text { KF2863 } \\
37\end{array}$ & $\begin{array}{l}\text { Li \& Cui } \\
\text { 2013a }\end{array}$ \\
\hline M. hengduanensis & Cui8176 & China & $\begin{array}{l}\text { JQ31437 } \\
0\end{array}$ & $\begin{array}{l}\text { KX9006 } \\
97\end{array}$ & $\begin{array}{l}\text { KX9007 } \\
49\end{array}$ & $\begin{array}{l}\text { MG8677 } \\
00\end{array}$ & $\begin{array}{l}\text { Li \& Cui } \\
2013 a\end{array}$ \\
\hline M. hexagonoides & Cui6592 & China & $\begin{array}{l}\text { JQ78040 } \\
2\end{array}$ & $\begin{array}{l}\text { JQ78043 } \\
8\end{array}$ & - & - & $\begin{array}{l}\text { Li \& Cui } \\
\text { 2013a }\end{array}$ \\
\hline M. hexagonoides & Cui13853 & China & $\begin{array}{l}\text { MW694 } \\
880\end{array}$ & $\begin{array}{l}\text { MW694 } \\
924\end{array}$ & $\begin{array}{l}\text { MW694 } \\
900\end{array}$ & $\begin{array}{l}\text { MZ6186 } \\
25\end{array}$ & $\begin{array}{l}\text { Present } \\
\text { study }\end{array}$ \\
\hline M. major & Cui10253 & China & $\begin{array}{l}\text { JQ31436 } \\
6\end{array}$ & $\begin{array}{l}\text { JQ78043 } \\
7\end{array}$ & $\begin{array}{l}\text { MK1165 } \\
02\end{array}$ & - & $\begin{array}{l}\text { Li \& Cui } \\
2013 a\end{array}$ \\
\hline M. major & Yuan1183 & China & $\begin{array}{l}\text { JQ31436 } \\
5\end{array}$ & - & - & - & $\begin{array}{l}\text { Li \& Cui } \\
\text { 2013a }\end{array}$ \\
\hline M. rimosa & $\begin{array}{l}\text { Dai15357 } \\
\text { (Holotype) }\end{array}$ & China & $\begin{array}{l}\text { KY4494 } \\
36\end{array}$ & $\begin{array}{l}\text { KY4494 } \\
47\end{array}$ & $\begin{array}{l}\text { MW694 } \\
908\end{array}$ & - & $\begin{array}{l}\text { Yuan et al. } \\
2017\end{array}$ \\
\hline M. rimosa & Dai21997 & China & $\begin{array}{l}\text { MW422 } \\
262\end{array}$ & - & $\begin{array}{l}\text { MW694 } \\
909\end{array}$ & - & $\begin{array}{l}\text { Present } \\
\text { study }\end{array}$ \\
\hline M. tropica & Cui13740 & China & $\begin{array}{l}\text { KY4494 } \\
38\end{array}$ & $\begin{array}{l}\text { KY4494 } \\
49\end{array}$ & $\begin{array}{l}\text { MW694 } \\
910\end{array}$ & $\begin{array}{l}\text { MZ6186 } \\
29\end{array}$ & $\begin{array}{l}\text { Yuan et al. } \\
2017\end{array}$ \\
\hline M. tropica & $\begin{array}{l}\text { Cui13660 } \\
\text { (Holotype) }\end{array}$ & China & $\begin{array}{l}\text { KY4494 } \\
37\end{array}$ & $\begin{array}{l}\text { KY4494 } \\
48\end{array}$ & $\begin{array}{l}\text { MW694 } \\
911\end{array}$ & $\begin{array}{l}\text { MZ6186 } \\
30\end{array}$ & $\begin{array}{l}\text { Yuan et al. } \\
2017\end{array}$ \\
\hline M. violacea & $\begin{array}{l}\text { Cui6570 } \\
\text { (Holotype) }\end{array}$ & China & $\begin{array}{l}\text { JQ78039 } \\
3\end{array}$ & - & - & - & $\begin{array}{l}\text { Li \& Cui } \\
\text { 2013a }\end{array}$ \\
\hline
\end{tabular}


Table 2 Continued.

\begin{tabular}{|c|c|c|c|c|c|c|c|}
\hline \multirow[t]{2}{*}{ Species } & \multirow[t]{2}{*}{ Sample no. } & \multirow{2}{*}{$\begin{array}{l}\text { Geographic } \\
\text { origin }\end{array}$} & \multicolumn{4}{|c|}{ GenBank accessions } & \multirow[t]{2}{*}{ References } \\
\hline & & & ITS & nLSU & mtSSU & TEF & \\
\hline M. violacea & Cui13845 & China & $\begin{array}{l}\text { MG8472 } \\
11\end{array}$ & $\begin{array}{l}\text { MG8472 } \\
20\end{array}$ & $\begin{array}{l}\text { MG8472 } \\
32\end{array}$ & $\begin{array}{l}\text { MG8677 } \\
03\end{array}$ & $\begin{array}{l}\text { Cui et al. } \\
2019\end{array}$ \\
\hline M. violacea & Cui13838 & China & $\begin{array}{l}\text { MG8472 } \\
10\end{array}$ & $\begin{array}{l}\text { MG8472 } \\
19\end{array}$ & $\begin{array}{l}\text { MG8472 } \\
31\end{array}$ & $\begin{array}{l}\text { MG8677 } \\
02\end{array}$ & $\begin{array}{l}\text { Cui et al. } \\
2019\end{array}$ \\
\hline M. yunnanensis & Cui12614A & China & $\begin{array}{l}\text { KY4494 } \\
42\end{array}$ & $\begin{array}{l}\text { KY4494 } \\
53\end{array}$ & $\begin{array}{l}\text { MW694 } \\
922\end{array}$ & $\begin{array}{l}\text { MZ6186 } \\
28\end{array}$ & $\begin{array}{l}\text { Yuan et al. } \\
2017\end{array}$ \\
\hline M. yunnanensis & $\begin{array}{l}\text { Dai13870 } \\
\text { (Holotype) }\end{array}$ & China & $\begin{array}{l}\text { KY4494 } \\
43\end{array}$ & $\begin{array}{l}\text { KY4494 } \\
54\end{array}$ & $\begin{array}{l}\text { MW694 } \\
907\end{array}$ & - & $\begin{array}{l}\text { Yuan et al. } \\
2017\end{array}$ \\
\hline $\begin{array}{l}\text { Megasporoporia } \\
\text { bannaensis }\end{array}$ & $\begin{array}{l}\text { Dai12306 } \\
\text { (Holotype) }\end{array}$ & China & $\begin{array}{l}\text { JQ31436 } \\
2\end{array}$ & $\begin{array}{l}\text { JQ31437 } \\
9\end{array}$ & - & - & $\begin{array}{l}\text { Li \& Cui } \\
\text { 2013a }\end{array}$ \\
\hline M. bannaensis & Dai13596 & China & $\begin{array}{l}\text { KX9006 } \\
53\end{array}$ & $\begin{array}{l}\text { KX9007 } \\
02\end{array}$ & $\begin{array}{l}\text { KX9007 } \\
54\end{array}$ & $\begin{array}{l}\text { KX9008 } \\
38\end{array}$ & $\begin{array}{l}\text { Cui et al. } \\
2019\end{array}$ \\
\hline M. cavernulosa & JV0904/52J & USA & $\begin{array}{l}\text { JF89410 } \\
7\end{array}$ & - & - & - & \\
\hline M. cavernulosa & JV0904/50J & USA & $\begin{array}{l}\text { JF89410 } \\
5\end{array}$ & - & - & - & \\
\hline M. cavernulosa & JV0904/81 & USA & $\begin{array}{l}\text { MW989 } \\
395\end{array}$ & - & - & - & $\begin{array}{l}\text { Present } \\
\text { study }\end{array}$ \\
\hline M. inflata & Dai17882 & Malaysia & $\begin{array}{l}\text { MW694 } \\
886\end{array}$ & $\begin{array}{l}\text { MW694 } \\
929\end{array}$ & $\begin{array}{l}\text { MW694 } \\
914\end{array}$ & - & $\begin{array}{l}\text { Present } \\
\text { study }\end{array}$ \\
\hline M. inflata & $\begin{array}{l}\text { Dai17478 } \\
\text { (Holotype) }\end{array}$ & Malaysia & $\begin{array}{l}\text { MW694 } \\
887\end{array}$ & $\begin{array}{l}\text { MW694 } \\
930\end{array}$ & $\begin{array}{l}\text { MW694 } \\
915\end{array}$ & $\begin{array}{l}\text { MZ6186 } \\
33\end{array}$ & $\begin{array}{l}\text { Present } \\
\text { study }\end{array}$ \\
\hline M. mexicana & JV1806/4J & Honduras & $\begin{array}{l}\text { MW989 } \\
396\end{array}$ & - & - & - & $\begin{array}{l}\text { Present } \\
\text { study }\end{array}$ \\
\hline M. minor & Dai18322 & Vietnam & $\begin{array}{l}\text { MW694 } \\
881\end{array}$ & $\begin{array}{l}\text { MW694 } \\
925\end{array}$ & $\begin{array}{l}\text { MW694 } \\
901\end{array}$ & $\begin{array}{l}\text { MZ6186 } \\
24\end{array}$ & $\begin{array}{l}\text { Present } \\
\text { study }\end{array}$ \\
\hline M. minor & $\begin{array}{l}\text { Dai12170 } \\
\text { (Holotype) }\end{array}$ & China & $\begin{array}{l}\text { JQ31436 } \\
3\end{array}$ & $\begin{array}{l}\text { JQ31438 } \\
0\end{array}$ & $\begin{array}{l}\text { MW694 } \\
902\end{array}$ & $\begin{array}{l}\text { KF4949 } \\
80\end{array}$ & $\begin{array}{l}\text { Li \& Cui } \\
2013 a\end{array}$ \\
\hline M. minuta & Zhou120 & China & $\begin{array}{l}\text { JX16305 } \\
5\end{array}$ & $\begin{array}{l}\text { JX16305 } \\
6\end{array}$ & $\begin{array}{l}\text { KF2183 } \\
36\end{array}$ & - & \\
\hline M. minuta & Cui13945 & China & $\begin{array}{l}\text { MW989 } \\
397\end{array}$ & $\begin{array}{l}\text { MW965 } \\
596\end{array}$ & - & - & $\begin{array}{l}\text { Present } \\
\text { study }\end{array}$ \\
\hline M. setulosa & JV1008_51J & USA & $\begin{array}{l}\text { JF89410 } \\
9\end{array}$ & - & - & - & $\begin{array}{l}\text { Li \& Cui } \\
2013 a\end{array}$ \\
\hline M. setulosa & JV1008_102J & USA & $\begin{array}{l}\text { JF89411 } \\
0\end{array}$ & - & - & - & $\begin{array}{l}\text { Li \& Cui } \\
\text { 2013a }\end{array}$ \\
\hline $\begin{array}{l}\text { Megasporoporiella } \\
\text { australiae }\end{array}$ & $\begin{array}{l}\text { Dai18657 } \\
\text { (Holotype) }\end{array}$ & Australia & $\begin{array}{l}\text { MW694 } \\
888\end{array}$ & $\begin{array}{l}\text { MW694 } \\
931\end{array}$ & $\begin{array}{l}\text { MW694 } \\
916\end{array}$ & $\begin{array}{l}\text { MZ6186 } \\
34\end{array}$ & $\begin{array}{l}\text { Present } \\
\text { study }\end{array}$ \\
\hline M. australiae & Dai18658 & Australia & $\begin{array}{l}\text { MW694 } \\
889\end{array}$ & $\begin{array}{l}\text { MW694 } \\
932\end{array}$ & $\begin{array}{l}\text { MW694 } \\
917\end{array}$ & $\begin{array}{l}\text { MZ6186 } \\
35\end{array}$ & $\begin{array}{l}\text { Present } \\
\text { study }\end{array}$ \\
\hline M. hubeiensis & Dai18102 & China & $\begin{array}{l}\text { MW694 } \\
890\end{array}$ & $\begin{array}{l}\text { MW694 } \\
933\end{array}$ & $\begin{array}{l}\text { MW694 } \\
918\end{array}$ & $\begin{array}{l}\text { MZ6186 } \\
36\end{array}$ & $\begin{array}{l}\text { Present } \\
\text { study }\end{array}$ \\
\hline M. hubeiensis & Dai18103 & China & $\begin{array}{l}\text { MW694 } \\
891\end{array}$ & $\begin{array}{l}\text { MW694 } \\
934\end{array}$ & $\begin{array}{l}\text { MW694 } \\
919\end{array}$ & - & $\begin{array}{l}\text { Present } \\
\text { study }\end{array}$ \\
\hline M. hubeiensis & $\begin{array}{l}\text { Wei2045 } \\
\text { (Holotype) }\end{array}$ & China & $\begin{array}{l}\text { JQ78038 } \\
7\end{array}$ & $\begin{array}{l}\text { JQ78042 } \\
1\end{array}$ & $\begin{array}{l}\text { KX8383 } \\
96\end{array}$ & - & $\begin{array}{l}\text { Cui et al. } \\
2019\end{array}$ \\
\hline M. pseudocavernulosa & $\begin{array}{l}\text { Yuan1270 } \\
\text { (Holotype) }\end{array}$ & China & $\begin{array}{l}\text { JQ31436 } \\
0\end{array}$ & $\begin{array}{l}\text { JQ31439 } \\
4\end{array}$ & - & - & $\begin{array}{l}\text { Li \& Cui } \\
\text { 2013a }\end{array}$ \\
\hline M. pseudocavernulosa & Dai19379 & China & $\begin{array}{l}\text { MW694 } \\
882\end{array}$ & - & $\begin{array}{l}\text { MW694 } \\
904\end{array}$ & $\begin{array}{l}\text { MZ6186 } \\
26\end{array}$ & $\begin{array}{l}\text { Present } \\
\text { study }\end{array}$ \\
\hline M. rhododendri & $\begin{array}{l}\text { Dai4226 } \\
\text { (Holotype) }\end{array}$ & China & $\begin{array}{l}\text { JQ31435 } \\
6\end{array}$ & $\begin{array}{l}\text { JQ31439 } \\
2\end{array}$ & $\begin{array}{l}\text { MW694 } \\
905\end{array}$ & - & $\begin{array}{l}\text { Li \& Cui } \\
2013 a\end{array}$ \\
\hline M. rhododendri & Cui12432 & China & $\begin{array}{l}\text { MW694 } \\
883\end{array}$ & $\begin{array}{l}\text { MW694 } \\
927\end{array}$ & $\begin{array}{l}\text { MW694 } \\
906\end{array}$ & $\begin{array}{l}\text { MZ6186 } \\
27\end{array}$ & $\begin{array}{l}\text { Present } \\
\text { study }\end{array}$ \\
\hline
\end{tabular}


Table 2 Continued.

\begin{tabular}{|c|c|c|c|c|c|c|c|}
\hline \multirow[t]{2}{*}{ Species } & \multirow[t]{2}{*}{ Sample no. } & \multirow{2}{*}{$\begin{array}{l}\text { Geographic } \\
\text { origin }\end{array}$} & \multicolumn{4}{|c|}{ GenBank accessions } & \multirow[t]{2}{*}{ References } \\
\hline & & & ITS & nLSU & mtSSU & TEF & \\
\hline M. subcavernulosa & Cui9252 & China & $\begin{array}{l}\text { JQ78037 } \\
8\end{array}$ & $\begin{array}{l}\text { JQ78041 } \\
6\end{array}$ & $\begin{array}{l}\text { MG8472 } \\
35\end{array}$ & $\begin{array}{l}\text { MG8677 } \\
06\end{array}$ & $\begin{array}{l}\text { Li \& Cui } \\
2013 a\end{array}$ \\
\hline M. subcavernulosa & Cui14247 & China & $\begin{array}{l}\text { MG8472 } \\
13\end{array}$ & $\begin{array}{l}\text { MG8472 } \\
22\end{array}$ & $\begin{array}{l}\text { MG8472 } \\
34\end{array}$ & $\begin{array}{l}\text { MG8677 } \\
05\end{array}$ & $\begin{array}{l}\text { Cui et al. } \\
2019\end{array}$ \\
\hline $\begin{array}{l}\text { Neodatronia } \\
\text { gaoligongensis }\end{array}$ & Cui8055 & China & $\begin{array}{l}\text { JX55926 } \\
9\end{array}$ & $\begin{array}{l}\text { JX55928 } \\
6\end{array}$ & $\begin{array}{l}\text { MG8472 } \\
36\end{array}$ & $\begin{array}{l}\text { KX9008 } \\
46\end{array}$ & $\begin{array}{l}\text { Li et al. } \\
2014\end{array}$ \\
\hline N. gaoligongensis & Cui8186 & China & $\begin{array}{l}\text { JX55926 } \\
8\end{array}$ & $\begin{array}{l}\text { JX55928 } \\
5\end{array}$ & $\begin{array}{l}\text { MG8472 } \\
37\end{array}$ & - & $\begin{array}{l}\text { Li et al. } \\
2014\end{array}$ \\
\hline Perenniporia martia & Cui4055 & China & $\begin{array}{l}\text { KX9006 } \\
41\end{array}$ & $\begin{array}{l}\text { KX9006 } \\
88\end{array}$ & $\begin{array}{l}\text { KX9007 } \\
37\end{array}$ & - & $\begin{array}{l}\text { Cui et al. } \\
2019\end{array}$ \\
\hline P. martia & Cui7992 & China & $\begin{array}{l}\text { HQ8766 } \\
03\end{array}$ & $\begin{array}{l}\text { HQ6541 } \\
14\end{array}$ & $\begin{array}{l}\text { KF0510 } \\
41\end{array}$ & $\begin{array}{l}\text { KF1811 } \\
35\end{array}$ & $\begin{array}{l}\text { Zhao \& Cui } \\
2012\end{array}$ \\
\hline Polyporus arcularius & Cui10998 & China & $\begin{array}{l}\text { KX5489 } \\
73\end{array}$ & $\begin{array}{l}\text { KX5489 } \\
95\end{array}$ & $\begin{array}{l}\text { KX5490 } \\
29\end{array}$ & $\begin{array}{l}\text { KX5490 } \\
59\end{array}$ & $\begin{array}{l}\text { Zhou \& Cui } \\
2017\end{array}$ \\
\hline P. arcularius & Cui11398 & China & $\begin{array}{l}\text { KU1897 } \\
66\end{array}$ & $\begin{array}{l}\text { KU1897 } \\
97\end{array}$ & $\begin{array}{l}\text { KU1899 } \\
47\end{array}$ & $\begin{array}{l}\text { KU1899 } \\
11\end{array}$ & $\begin{array}{l}\text { Zhou et al. } \\
2016\end{array}$ \\
\hline $\begin{array}{l}\text { P. megasporoporus } \\
\text { (Megasporoporiella } \\
\text { lacerata) }\end{array}$ & $\begin{array}{l}\text { Yuan3880 } \\
\text { (Holotype) }\end{array}$ & China & $\begin{array}{l}\text { JQ31437 } \\
7\end{array}$ & $\begin{array}{l}\text { JQ31439 } \\
5\end{array}$ & - & $\begin{array}{l}\text { KF2863 } \\
34\end{array}$ & $\begin{array}{l}\text { Li \& Cui } \\
2013 \mathrm{a}\end{array}$ \\
\hline $\begin{array}{l}\text { P. megasporoporus } \\
\text { (Megasporoporiella } \\
\text { lacerata) }\end{array}$ & Yuan3874 & China & - & $\begin{array}{l}\text { MW694 } \\
926\end{array}$ & $\begin{array}{l}\text { MW694 } \\
903\end{array}$ & - & $\begin{array}{l}\text { Present } \\
\text { study }\end{array}$ \\
\hline P. tuberaster & Dai12462 & China & $\begin{array}{l}\text { KU5075 } \\
80\end{array}$ & $\begin{array}{l}\text { KU5075 } \\
82\end{array}$ & $\begin{array}{l}\text { KU5075 } \\
84\end{array}$ & $\begin{array}{l}\text { KU5075 } \\
90\end{array}$ & $\begin{array}{l}\text { Zhou et al. } \\
2016\end{array}$ \\
\hline P. tuberaster & Dai11271 & China & $\begin{array}{l}\text { KU1897 } \\
69\end{array}$ & $\begin{array}{l}\text { KU1898 } \\
00\end{array}$ & $\begin{array}{l}\text { KU1899 } \\
50\end{array}$ & $\begin{array}{l}\text { KU1899 } \\
14\end{array}$ & $\begin{array}{l}\text { Zhou et al. } \\
2016\end{array}$ \\
\hline P. varius & Cui12249 & China & $\begin{array}{l}\text { KU5075 } \\
81\end{array}$ & $\begin{array}{l}\text { KU5075 } \\
83\end{array}$ & $\begin{array}{l}\text { KU5075 } \\
85\end{array}$ & $\begin{array}{l}\text { KU5075 } \\
91\end{array}$ & $\begin{array}{l}\text { Zhou et al. } \\
2016\end{array}$ \\
\hline P. varius & Dai13874 & China & $\begin{array}{l}\text { KU1897 } \\
77\end{array}$ & $\begin{array}{l}\text { KU1898 } \\
08\end{array}$ & $\begin{array}{l}\text { KU1899 } \\
58\end{array}$ & $\begin{array}{l}\text { KU1899 } \\
23\end{array}$ & $\begin{array}{l}\text { Zhou et al. } \\
2016\end{array}$ \\
\hline Trametes hirsuta & RLG5133T & USA & $\begin{array}{l}\text { JN16494 } \\
1\end{array}$ & $\begin{array}{l}\text { JN16480 } \\
1\end{array}$ & - & $\begin{array}{l}\text { JN16489 } \\
1\end{array}$ & $\begin{array}{l}\text { Li \& Cui } \\
2013 a\end{array}$ \\
\hline T. ochracea & HHB13445sp & USA & $\begin{array}{l}\text { JN16495 } \\
4\end{array}$ & $\begin{array}{l}\text { JN16481 } \\
2\end{array}$ & - & $\begin{array}{l}\text { JN16490 } \\
4\end{array}$ & $\begin{array}{l}\text { Li \& Cui } \\
\text { 2013a }\end{array}$ \\
\hline
\end{tabular}

New species and sequences are shown in bold

\section{Phylogenetic analysis}

Sequences generated in this study were aligned with additional sequences downloaded from GenBank (Table 2) using Clustal X (Thompson et al. 1997) and BioEdit (Hall 1999). The data matrixes were edited in Mesquite v3.04 software (Maddison \& Maddison 2009). Sequence alignment was deposited at TreeBase (submission ID 28606 and 28610). Previous to phylogenetic analysis, ambiguous sequences at the start and the end were deleted and gaps were manually adjusted to optimize the alignment using BioEdit (Hall 1999). Two combined matrixes were reconstructed for phylogenetic analyses as a 2-gene dataset (ITS + nLSU) and a 4-gene dataset (ITS $+\mathrm{nLSU}+\mathrm{mtSSU}+\mathrm{TEF})$. The phylogenetic analyses used in this study followed the approach of Zhu et al. (2019) and Sun et al. (2020). Maximum parsimony (MP), Maximum likelihood (ML) and Bayesian inference (BI) were employed to perform phylogenetic analysis.

Sequences of Trametes hirsuta (Wulfen) Lloyd and T. ochracea (Pers.) Gilb. \& Ryvarden were used as outgroups to root trees. All characters were equally weighted and gaps were treated as missing data. Trees were inferred using heuristic search option with TBR branch swapping and 1,000 random sequence additions. Max-trees were set to 5,000, branches of zero length were collapsed and all parsimonious trees were saved. Clade robustness was assessed using bootstrap analysis with 1,000 replicates (Felsenstein 1985). Descriptive tree statistics tree length (TL), 
consistency index $(\mathrm{CI})$, retention index $(\mathrm{RI})$, rescaled consistency index (RC), and homoplasy index (HI) were calculated for each maximum parsimonious tree generated (Farris 1989, Farris et al. 1994, Swofford 2002, Yuan et al. 2017).

Maximum likelihood (ML) research was conducted with RAxML-HPC v. 8.2.3 (Stamatakis 2014) involved 1000 ML searches under the GTRGAMMA model, and only the maximum likelihood best tree from all searches was kept. In addition, 1000 rapid bootstrap replicates were run with the GTRCAT model to assess ML bootstrap values (ML) of the nodes.

MrMODELTEST 2.3 (Posada \& Crandall 1998, Nylander 2004) also was used to determine the best-fit evolution model for the combined dataset of ITS + nLSU and ITS + nLSU + mtSSU + TEF sequences for estimating Bayesian inference (BI). Bayesian inference was calculated with MrBayes 3.1.2 (Ronquist \& Huelsenbeck 2003). Four Markov chains were run for 2 runs from random starting trees for 2 million generations until the split deviation frequency value $<0.01$, and sampled every 100 generations. The first one-fourth sampled three were discarded as burn-in, while the remaining ones were used to calculate Bayesian posterior probabilities (BPP) of the clades.

Branches that received bootstrap support for Maximum parsimony (MP), Maximum Likelihood (ML), and Bayesian posterior probabilities (BPP) more than or equal to $50 \%$ (MP and ML) and 0.90 (BPP) were considered as significantly supported. (Figs 1-2). Phylogenetic trees were visualized with the program FigTree v. 1.4 .3 (http://tree.bio.ed.ac.uk/software/figtree/).

\section{Results}

\section{Phylogenetic analyses}

The consequence of phylogeny includes the combined dataset of ITS and nLSU sequences from 96 fungal collections representing 49 species. The dataset has an aligned length of 2074 characters, of which 1148 characters are constant, and 591 are parsimony-informative. MP analysis yields a tree $(\mathrm{TL}=2588, \mathrm{CI}=0.388, \mathrm{RI}=0.795, \mathrm{RC}=0.309, \mathrm{HI}=0.612)$. The best-fit evolutionary model is selected by maximum parsimony (MP) from the combined dataset of ITS + nLSU sequences. The MP and ML values $(\geq 50 \%)$ and BPP $(\geq 0.90)$ are shown at the nodes. So, the topology from the MP tree is presented along with statistical values from the MP/ML/BPP algorithms (Fig. 1).

The consequence of phylogeny includes the combined dataset of ITS, nLSU, mtSSU, and TEF sequences from 94 fungal collections representing 48 species. The dataset has an aligned length of 3363 characters, of which 2177 characters are constant, and 1093 are parsimonyinformative. $\mathrm{MP}$ analysis yields a tree $(\mathrm{TL}=4864, \mathrm{CI}=0.409, \mathrm{RI}=0.772, \mathrm{RC}=0.316, \mathrm{HI}=$ 0.591). The best-fit evolutionary model is selected by maximum parsimony (MP) from the combined dataset of ITS + nLSU + mtSSU + TEF sequences. The MP and ML values $(\geq 50 \%)$ and BPP $(\geq 0.90)$ are shown at the nodes. So, the topology from the MP tree is presented along with statistical values from the MP/ML/BPP algorithms (Fig. 2).

Our phylogenies support previous conclusions (Li \& Cui 2013a, Yuan et al. 2017), three clades are formed in the topology (Fig. 1): Megasporia (79\% MP, 99\% ML, 1.00 BPP), Megasporoporia sensu stricto (91\% MP, 99\% ML, $1.00 \mathrm{BPP}$ ) and Megasporoporiella (95\% MP, $100 \%$ ML, 1.00 BPP).

The Megasporia clade includes twelve species: $M$. bambusae sp. nov., M. cystidiolophora B.K. Cui \& Hai J. Li, M. ellipsoidea B.K. Cui \& Hai J. Li, M. fusiformis sp. nov., M. guangdongensis B.K. Cui \& Hai J. Li, M. hengduanensis B.K. Cui \& Hai J. Li, M. hexagonoides B.K. Cui et al., M. major B.K. Cui \& Hai J. Li, M. rimosa Y. Yuan et al., M. tropica Y. Yuan et al., M. violacea B.K. Cui et al. and M. yunnanensis Y. Yuan et al.

The Megasporoporia sensu stricto clade includes four species: M. bannaensis B.K. Cui \& Hai J. Li, M. inflata sp. nov., M. minor B.K, Cui \& Hai J. Li, and M. setulosa Rajchenb.

The Megasporoporiella clade includes five species: M. australiae sp. nov., M. hubeiensis comb. nov., M. pseudocavernulosa B.K. Cui \& Hai J. Li, M. rhododendri B.K. Cui \& Hai J. Li, and M. subcavernulosa B.K. Cui \& Hai J. Li. 
In addition, another clade (the unnamed clade of Megasporoporia sensu lato, Figs 1-2) includes three species: Dichomitus amazonicus Gomes-Silva et al., Megasporoporia cavernulosa (Berk.) Ryvarden and M. mexicana Ryvarden.

Four new species, Megasporia bambusae, Megasporia fusiformis, Megasporoporia inflata, Megasporoporiella australiae, and a new combination, Megasporoporiella hubeiensis, formed well-supported phylogenetic lineages (100\% ML, 100\% ML, 1.00 BPP) distinct from other known lineages (species) of Megasporoporia sensu lato (Fig. 2).

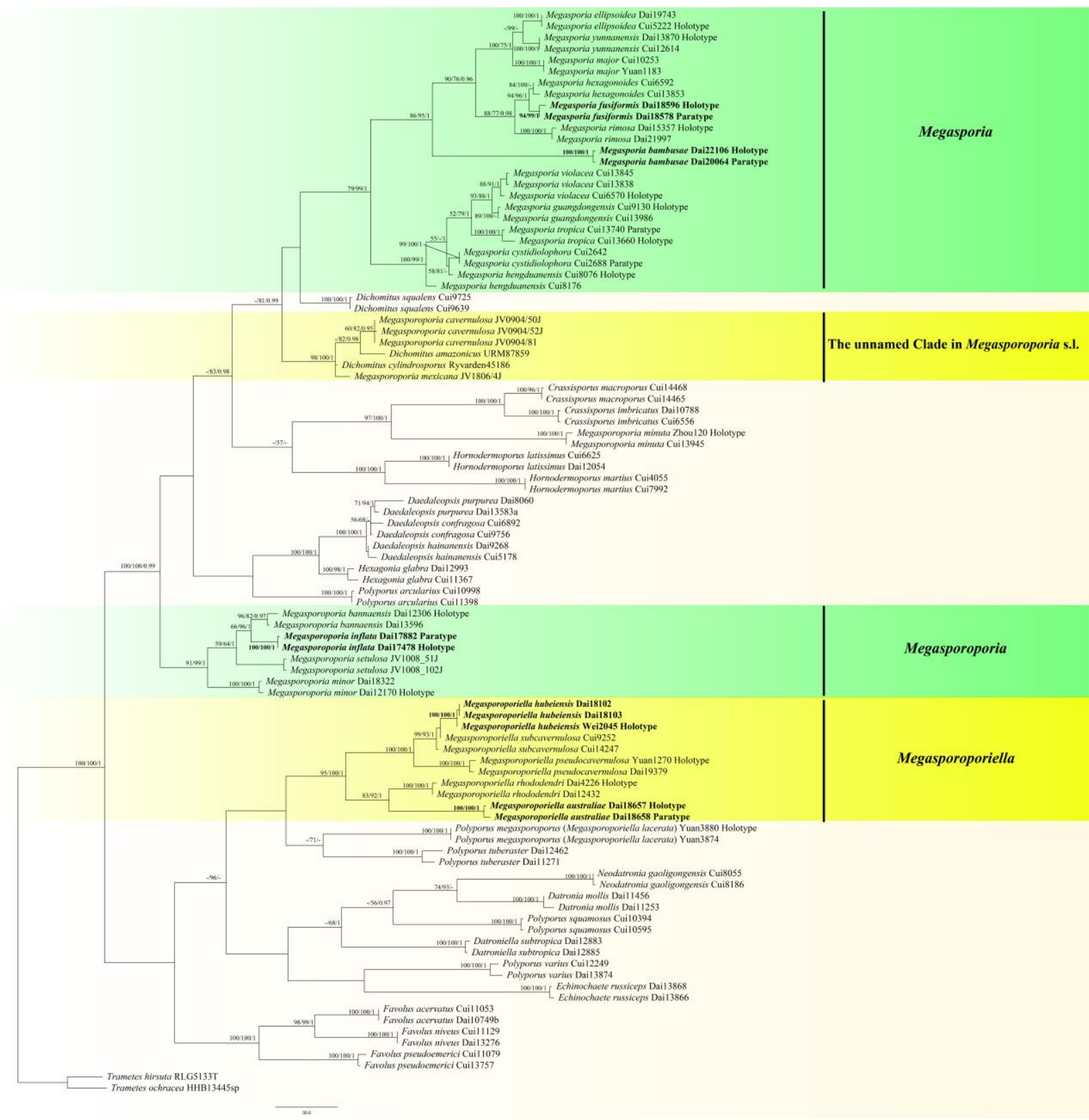

Figure 1 - Phylogeny of Megasporoporia sensu lato and related species generated by Maximum Parsimony based on combined ITS + nLSU sequences. Bootstrap supports for Maximum parsimony (MP), Maximum Likelihood (ML) and Bayesian posterior probabilities (BPP) were not lower than: 50\% (MP and ML) and 0.90 (BPP) on the branches. The new species and combination were in bold. 


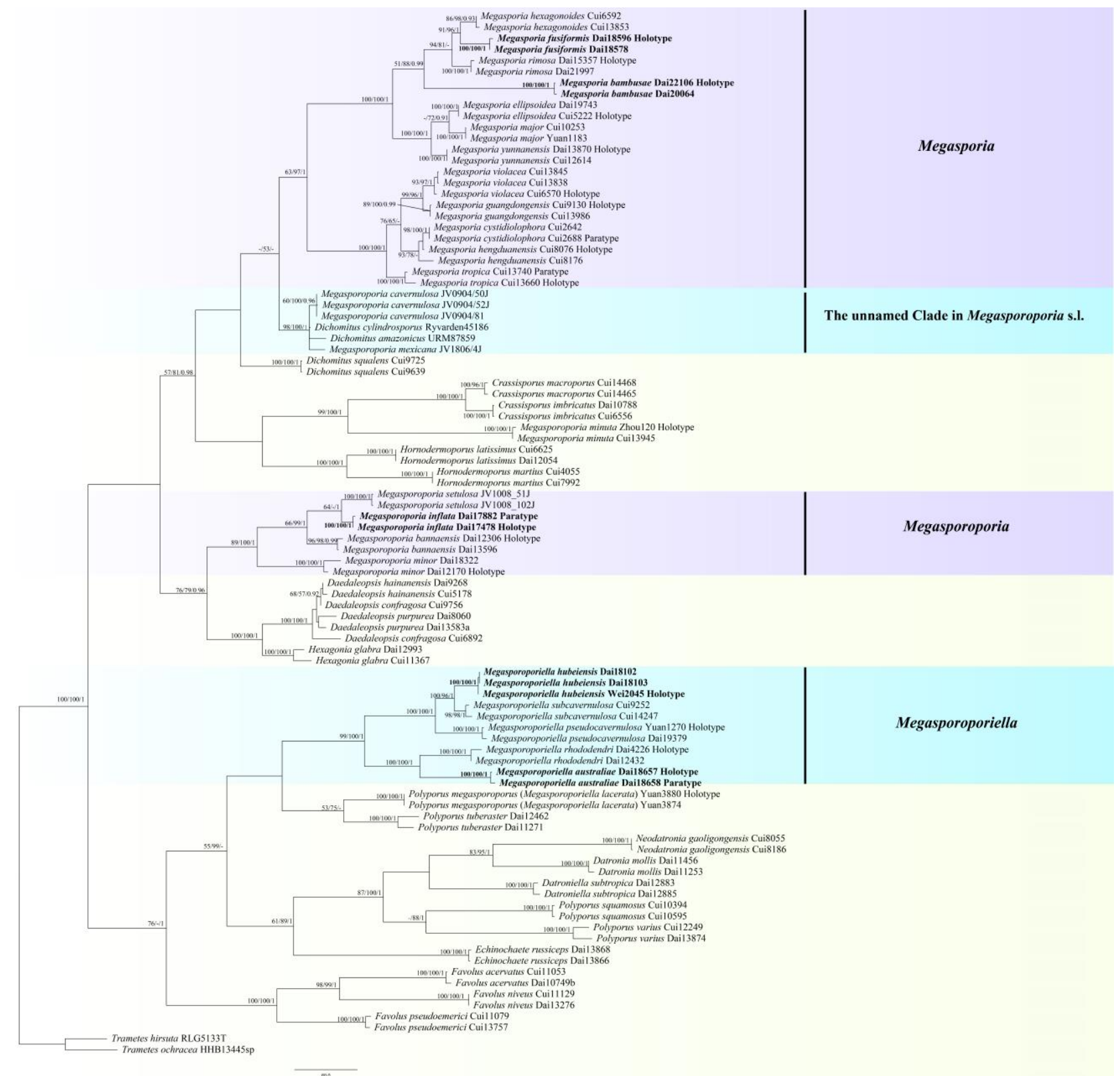

Figure 2 - Phylogeny of Megasporoporia sensu lato and related species generated by Maximum Parsimony based on combined ITS + nLSU + mtSSU + TEF sequences. Bootstrap supports for MP, ML and BPP were not lower than: $50 \%$ (MP and ML) and 0.90 (BPP) on the branches. The new species and combination were in bold.

\section{Taxonomy}

Megasporia bambusae Y.C. Dai, Yuan Yuan \& Ya. R. Wang sp. nov.

Figs 3-4 Index Fungorum number: IF558811; Facesoffungi number: FoF10470

Etymology - bambusae (Lat.): referring to the species growing on bamboo.

Basidiocarps annual, resupinate, corky, without odor or taste when fresh, becoming hard corky upon drying, up to $2.7 \mathrm{~cm}$ long, $2.2 \mathrm{~cm}$ wide, and $0.2 \mathrm{~mm}$ thick at center; sterile margin distinct, white, up to $1 \mathrm{~mm}$ wide. Pore surface white to cream when fresh, cream to buff when dry; pores angular, 4-5 per mm; dissepiments thick, entire; subiculum pale buff, corky, up to $0.05 \mathrm{~mm}$ thick; tubes cream, paler than subiculum, corky, up to $0.15 \mathrm{~mm}$ long. Hyphal system dimitic; generative hyphae bearing clamp connections; skeletal hyphae weakly dextrinoid, $\mathrm{CB}+$; tissues unchanged in $\mathrm{KOH}$ (not dissolved). Subicular generative hyphae hyaline, thin-walled, occasionally branched, 1.5-1.8 $\mu \mathrm{m}$ in diam; skeletal hyphae dominant, thick-walled with a narrow to wide 
lumen, frequently branched, mostly flexuous, interwoven, $2.5-3 \mu \mathrm{m}$ in diam. Tramal generative hyphae hyaline, thin-walled, occasionally branched, 1.5-1.8 $\mu \mathrm{m}$ in diam; skeletal hyphae dominant, thick-walled with a narrow to medium lumen, frequently branched, mostly flexuous, interwoven, 1.5-2 $\mu \mathrm{m}$ in diam. Dendrohyphidia present. Hyphal pegs absent. Cystidia absent; cystidioles present, subulate or ventricose, thin-walled, smooth, 14-41 × 4.8-14.8 $\mu \mathrm{m}$. Basidia clavate to pearshaped, usually constricted in middle, with four sterigmata and a basal clamp connection, 20-35.2 $\times 10-14.8 \mu \mathrm{m}$; basidioles in shape similar to basidia, but distinctly smaller. Small tetrahedric or polyhedric crystals frequently present among hymenium. Basidiospores ellipsoid, hyaline, fairly thick-walled, smooth, IKI-, CB-, (10.5-)11.8-14(-14.8) × (5.5-)5.8-6.8(-7.5) $\mu \mathrm{m}, \mathrm{L}=12.67 \mu \mathrm{m}$, $\mathrm{W}=6.53 \mu \mathrm{m}, \mathrm{Q}=1.91-1.96(\mathrm{n}=90 / 3)$.

Known distribution - widespread in tropical and subtropical regions.

Materials examined - China, Hainan Prov., Haikou, Jinniuling Park, on dead bamboo, 18 Nov 2020, Y.C. Dai 22106 (BJFC035998, holotype), Y.C. Dai 22113 (BJFC036005); Guangxi Auto. Reg., Yulin, Guishan Forest Park, on dead bamboo, 3 Jul 2019, Y.C. Dai 20064 (BJFC031738).

Notes - Megasporia bambusae is found from the tropical zone of China. It is readily distinguished from other species in Megasporoporia sensu lato by its fairly thick-walled basidiospores and growing on bamboo, all other members of Megasporoporia sensu lato have thinwalled basidiospores and growing on dicotyledon. Megasporia bambusae resembles Megasporia cystidiolophora, Megasporia guangdongensis and Megasporoporiella rhododendri by the overlapped distribution and almost the same size of pore (3-5 per $\mathrm{mm}$ ), but the latter three species have thin-walled basidiospores and lacks dendrohyphidia. Phylogenetically, Megasporia bambusae is related to Megasporia ellipsoidea, M. yunnanensis, M. major, M. hexagonoides, M. fusiformis and $M$. rimosa (Figs 1-2), but the latter six species have thin-walled basidiospores and growing on another angiosperm rather than bamboo (Ryvarden et al. 1982, Yuan et al. 2017).

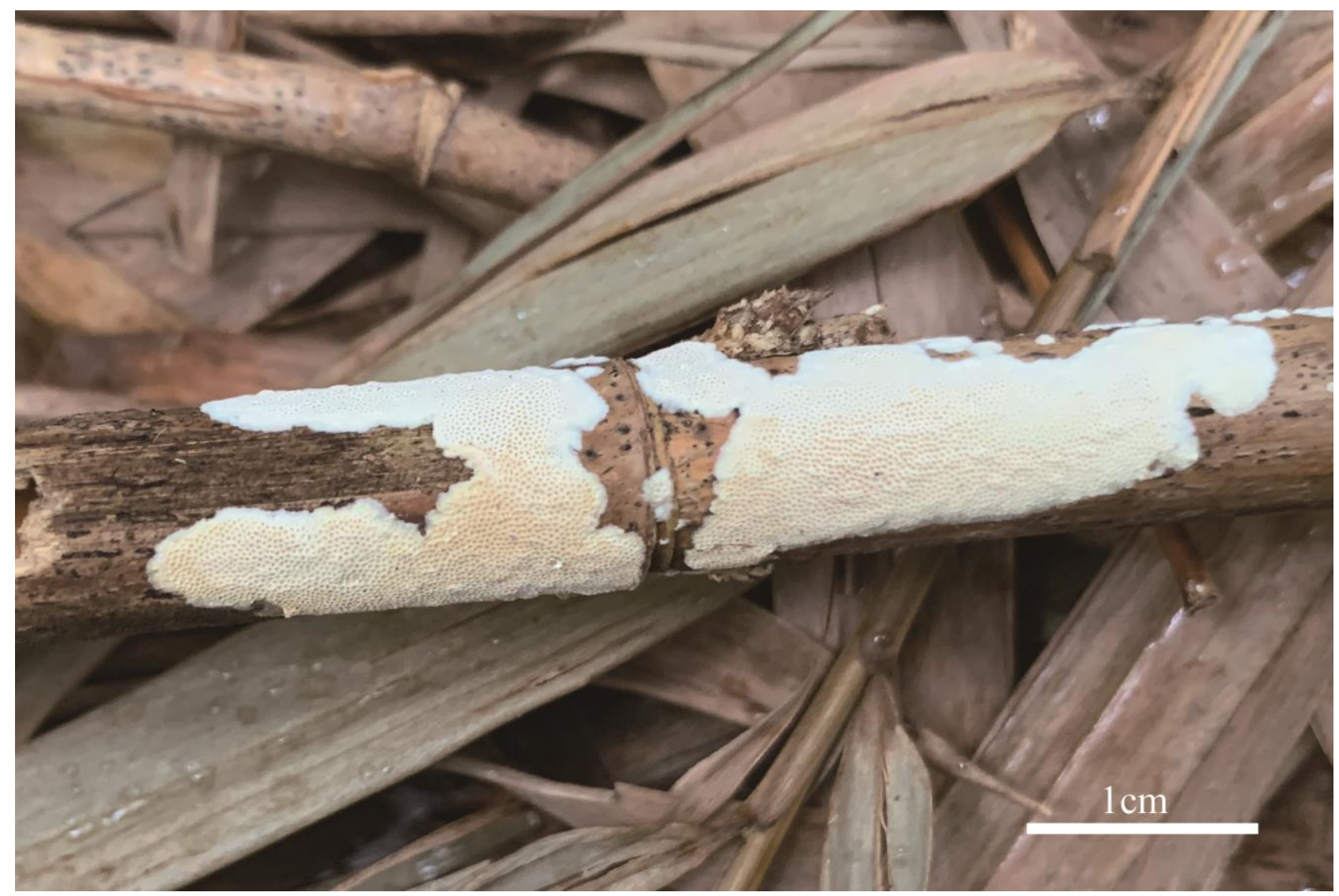

Figure 3 - Basidiocarps of Megasporia bambusae (the holotype, Dai 22106). 

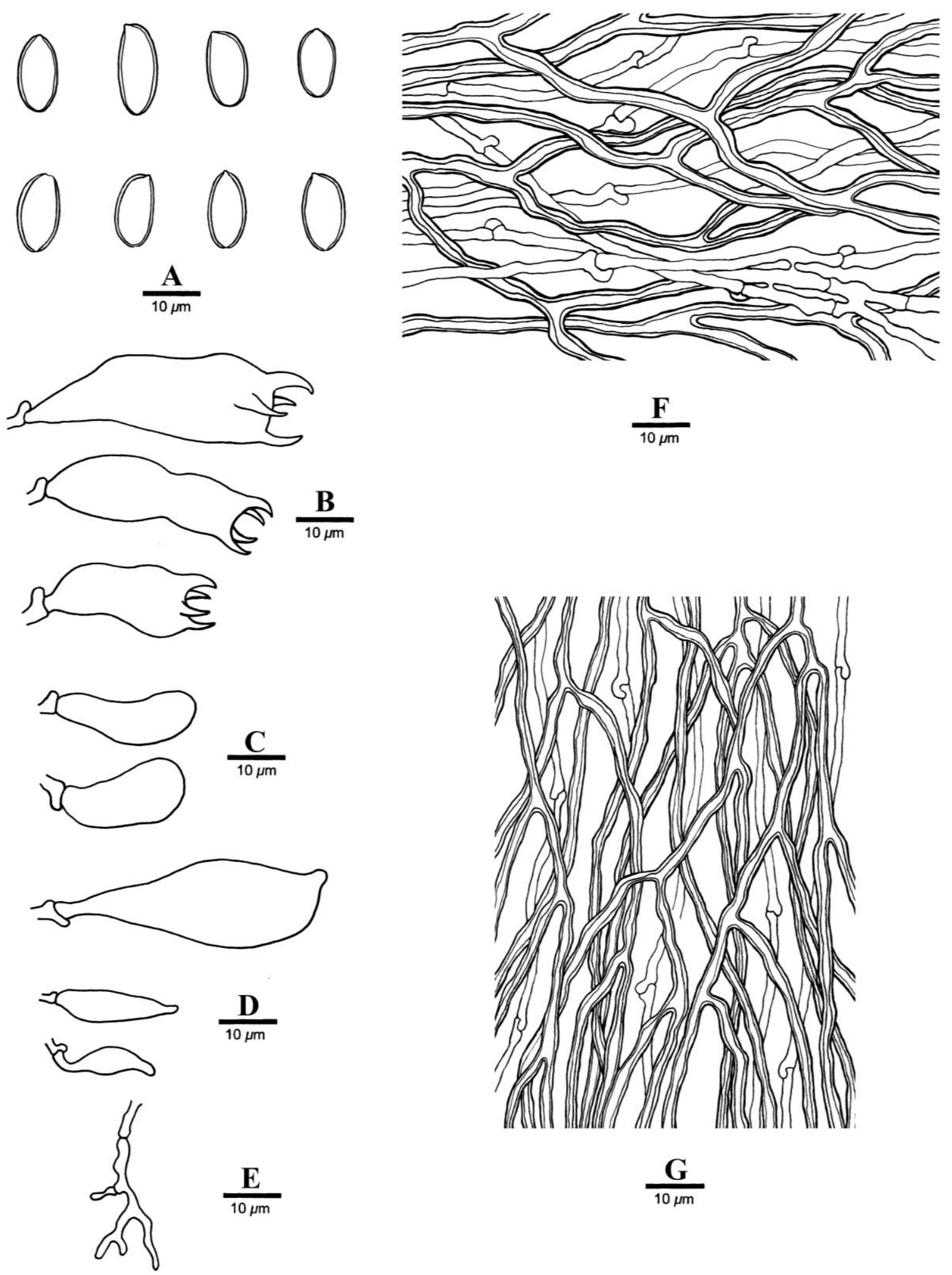

Figure 4 - Microscopic structures of Megasporia bambusae (drawn from the holotype, Dai 22106). A Basidiospores. B Basidia. C Basidioles. D Cystidioles. E Dendrohyphidia. E Hyphae from subiculum. G Hyphae from tubes.

Megasporia fusiformis Y.C. Dai, Yuan Yuan \& Ya.R. Wang, sp. nov.

Figs 5-6

Index Fungorum number: IF558812; Facesoffungi number: FoF10471

Etymology - fusiformis (Lat.): referring to the species producing fusiform basidiospores. 
Basidiocarps annual, resupinate, corky, without odor or taste when fresh, becoming hard corky when dry, up to $11.6 \mathrm{~cm}$ long, $2.2 \mathrm{~cm}$ wide, and $0.4 \mathrm{~mm}$ thick at center; sterile margin distinct, white, up to $1 \mathrm{~mm}$ wide. Pore surface cream when fresh, cream to buff-yellow when dry; pores angular, 3.5-4 per mm; dissepiments thick, entire; subiculum cream, corky, up to $0.2 \mathrm{~mm}$ thick; tubes cream, paler than subiculum, corky, up to $0.2 \mathrm{~mm}$ long. Hyphal system dimitic; generative hyphae bearing clamp connections; skeletal hyphae sometime simple septate, IKI-, $\mathrm{CB}+$; tissues unchanged in $\mathrm{KOH}$ (not dissolved). Subicular generative hyphae infrequent, hyaline, thin-walled, occasionally branched, 2-2.5 $\mu \mathrm{m}$ in diam; skeletal hyphae dominant, thick-walled with a narrow to wide lumen, frequently branched, occasionally simple septate, mostly flexuous, interwoven, $2.8-3 \mu \mathrm{m}$ in diam. Tramal generative thin-walled, occasionally branched, $2-3 \mu \mathrm{m}$ in diam; skeletal hyphae dominant, thick-walled with a narrow to medium lumen, frequently branched, mostly flexuous, interwoven, $2-3.5 \mu \mathrm{m}$ in diam. Dendrohyphidia present. Hyphal pegs absent. Cystidia absent; cystidioles present, ventricose, thin-walled, smooth, 23.2-28.5 × 5.2-9.5 $\mu \mathrm{m}$. Basidia clavate, usually constricted in middle, with four sterigmata and a basal clamp connection, 25.2-38.2 $\times 8.2-11.5 \mu \mathrm{m}$; basidioles in shape similar to basidia, but distinctly smaller. Small tetrahedric or polyhedric crystals frequently present among hymenium. Basidiospores fusiform, hyaline, thin-walled, smooth, sometimes with one or two guttules, IKI-, CB-, (14.1-)15$19.8(-20.2) \times(4-) 4.2-6.8(-7) \mu \mathrm{m}, \mathrm{L}=17.59 \mu \mathrm{m}, \mathrm{W}=5.12 \mu \mathrm{m}, \mathrm{Q}=3.24-3.68(\mathrm{n}=60 / 2)$.

Known distribution - widespread in subtropical regions.

Materials examined - Malaysia. Selangor, Kota Damansara, Community Forest Reserve, on rotten angiosperm wood, 16 April 2018, Y.C. Dai 18596 (BJFC026884, holotype), Y.C. Dai 18578 (BJFC026866).

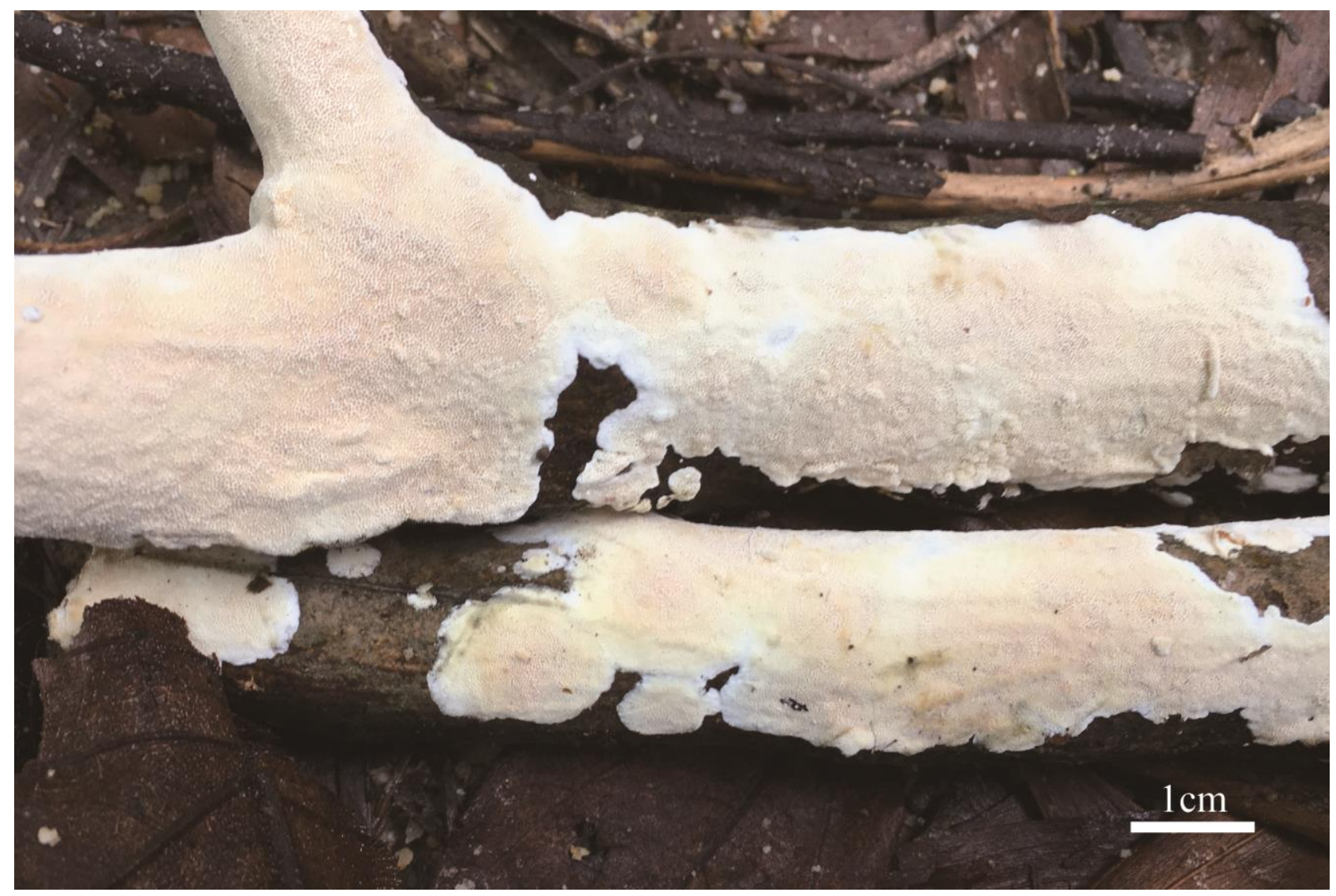

Figure 5 - Basidiocarps of Megasporia fusiformis (the holotype, Dai 18596).

Notes - Morphologically, Megasporia rimosa is similar to M. fusiformis by white to cream pore surface and extremely thin basidiocarp (less than $0.5 \mathrm{~mm}$ thick), but $M$. rimosa is different from $M$. fusiformis by its dextrinoid skeletal hyphae, cylindrical basidiospores and skeletal hyphae 
without any septa (Yuan et al. 2017). Phylogenetically $M$. fusiformis is closer to $M$. hexagonoides and $M$. rimosa (Figs 1-2), but $M$. hexagonoides differs from $M$. fusiformis by the bigger pores (0.51 per $\mathrm{mm}$ vs. 3.5-4 per $\mathrm{mm}$ ), the absence of dendrohyphidia, and skeletal hyphae without any septa (Dai \& Cui 2008).
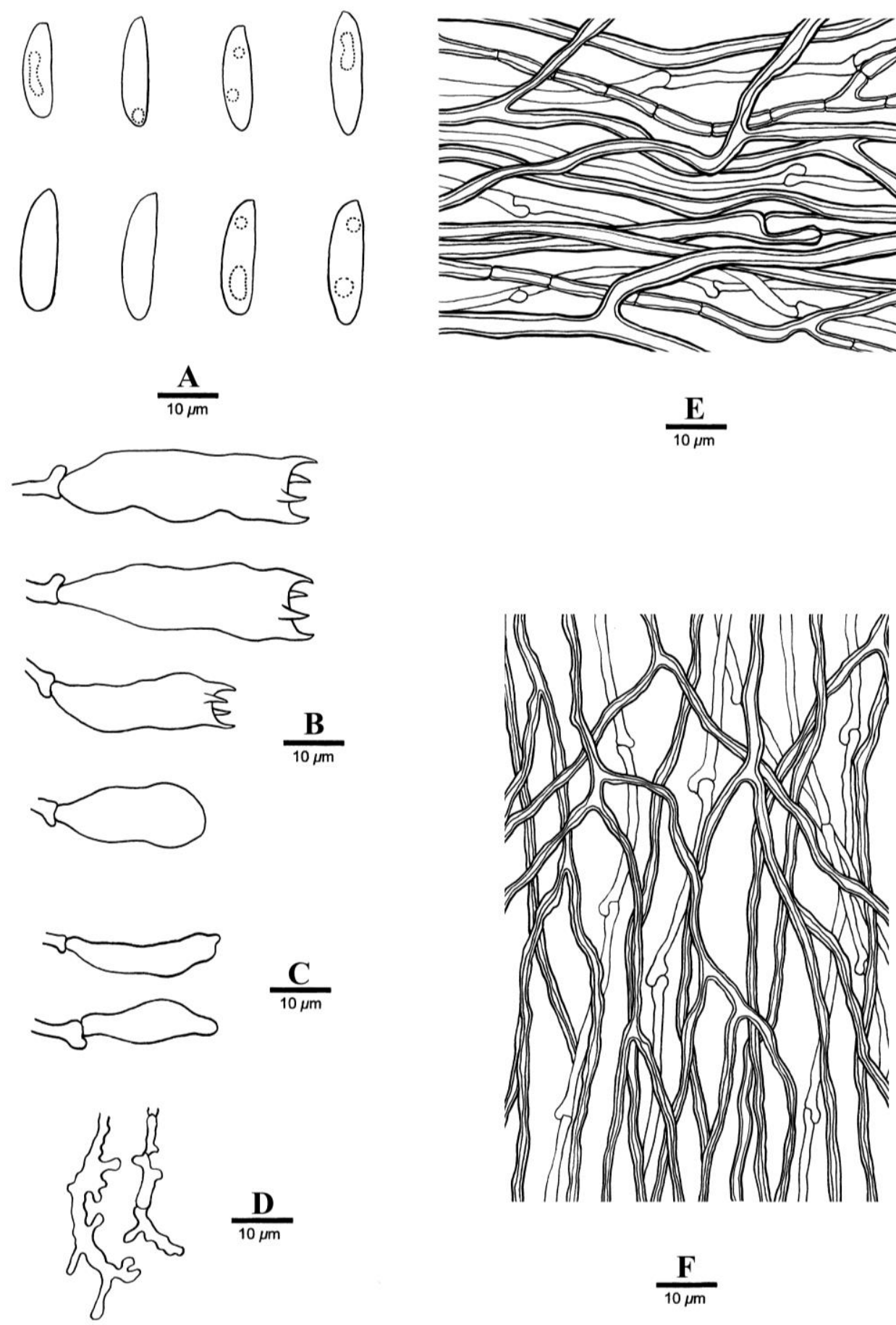

Figure 6 - Microscopic structures of Megasporia fusiformis (drawn from the holotype, Dai 18596). A Basidiospores. B Basidia and basidioles. C Cystidioles. D Dendrohyphidia. E Hyphae from subiculum. F Hyphae from tubes.

Megasporoporia inflata Y.C. Dai, Yuan Yuan \& Ya.R. Wang, sp. nov.

Figs $7-8$ Index Fungorum number: IF558813; Facesoffungi number: FoF10472 

$\mathrm{KOH}$.

Etymology - inflata (Lat.): referring to the skeletal hyphae of the species become swollen in

Basidiocarps annual, resupinate, cushion-shaped, corky, without odor or taste when fresh, becoming hard corky upon drying, up to $9.5 \mathrm{~cm}$ long, $2 \mathrm{~cm}$ wide, and $3.5 \mathrm{~mm}$ thick at center; sterile margin thinning out, cream to clay buff, up to $1 \mathrm{~mm}$ wide. Pore surface cream to buff when fresh, buff when dry; pores round to angular, 2.5-3 per mm; dissepiments thick, entire; subiculum buff, corky, up to $1 \mathrm{~mm}$ thick; tubes pale buff, corky, up to $2.5 \mathrm{~mm}$ long. Hyphal system dimitic; generative hyphae bearing clamp connections; skeletal hyphae strongly dextrinoid, CB+; tissues more or less dissolved and skeletal hyphae become strongly swollen in $\mathrm{KOH}$. Subicular generative hyphae infrequent, hyaline, thin-walled, moderately branched, mostly flexuous, $2-2.5 \mu \mathrm{m}$ in diam; skeletal hyphae dominant, thick-walled with a narrow to medium lumen, moderately branched, mostly flexuous, interwoven, $2-3.5 \mu \mathrm{m}$ in diam. Tramal generative hyaline, thin-walled, moderately branched, 2-2.5 $\mu \mathrm{m}$ in diam; skeletal hyphae dominant, thick-walled with a narrow lumen to subsolid, moderately branched, mostly flexuous, interwoven, 2.5-3 $\mu \mathrm{m}$ in diam. Dendrohyphidia absent. Hyphal pegs absent. Cystidia and cystidioles absent. Basidia broadly barrel-shaped to pyriform, with four sterigmata and a basal clamp connection, 14.2-22.5 $\times 7-8.9$ $\mu \mathrm{m}$; basidioles dominant in hymenium, in shape similar to basidia, but smaller. Big rhomboid or polyhedric crystals frequently present among hymenium. Basidiospores cylindrical, hyaline, thinwalled, smooth, with one big guttule, IKI-, CB-, (9.8-)10-11.8(-12) $\times 3.5-4.2 \mu \mathrm{m}, \mathrm{L}=10.38 \mu \mathrm{m}$, $\mathrm{W}=3.86 \mu \mathrm{m}, \mathrm{Q}=2.69-2.98(\mathrm{n}=60 / 2)$.

Known distribution - widespread in tropical and subtropical regions.

Materials examined - Malaysia. Selangor, Kota Damansara Community Forest Reserve, on fallen angiosperm twig, 19 June 2017, Y.C. Dai 17478 (BJFC025011, holotype). Singapore. Bukit, Timah Natural Reserve, on fallen angiosperm branch, 20 July 2017, Y.C. Dai 17882 (BJFC025414).

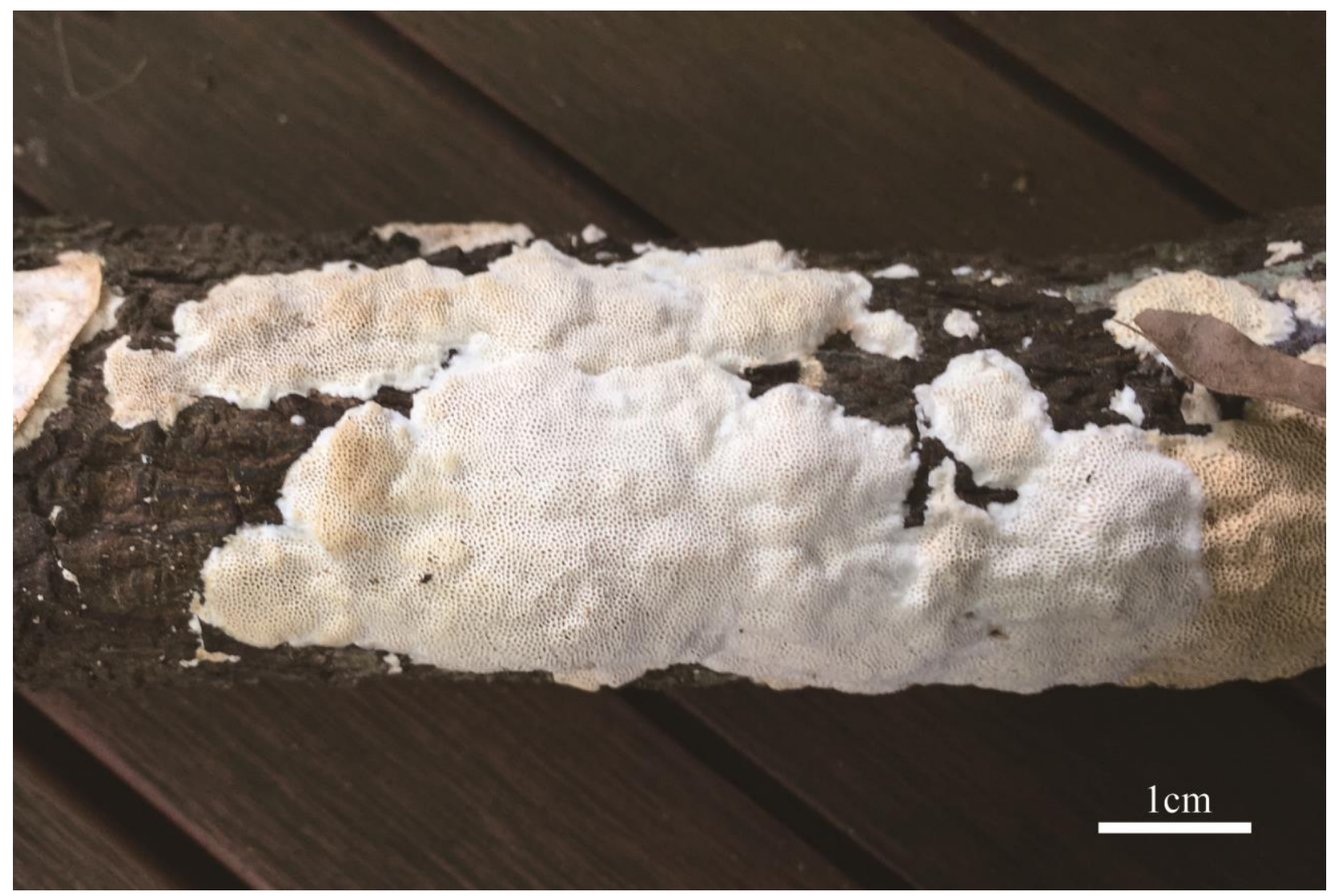

Figure 7 - Basidiocarps of Megasporoporia inflata (the holotype, Dai 17478). 

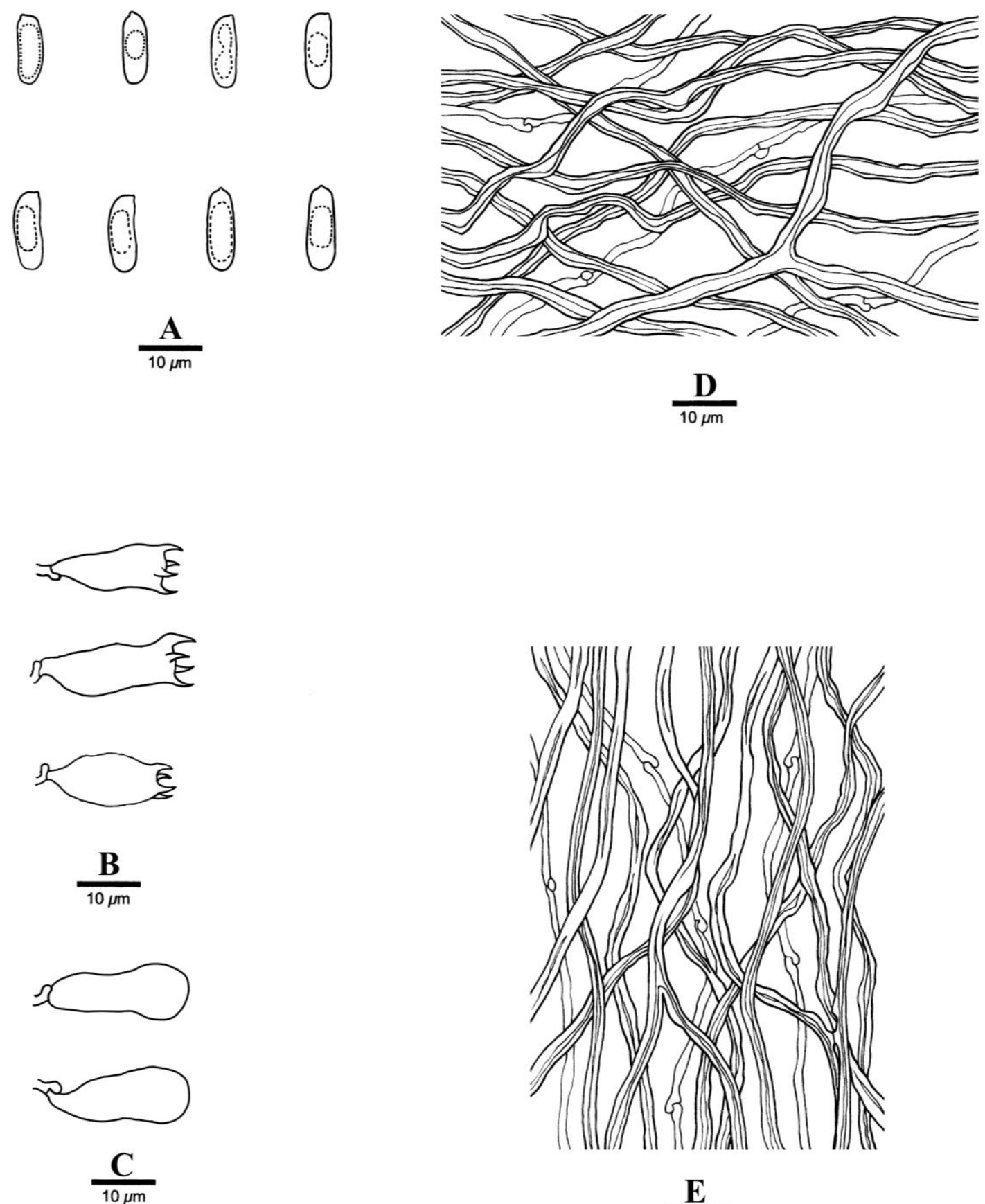

$\frac{\mathbf{E}}{10 \mu \mathrm{m}}$

Figure 8 - Microscopic structures of Megasporoporia inflata (drawn from the holotype, Dai 17478). A Basidiospores. B Basidia. C Basidioles. D Hyphae from subiculum. E Hyphae from tubes.

Notes - The skeletal hyphae of Megasporoporia inflata strongly swell in $\mathrm{KOH}$, and this feature is unique in Megasporoporia sensu lato. In addition, Megasporoporia inflata lacks hyphal pegs, cystidioles and dendrohyphidia. So, the above characteristics stand out the new species from other members of Megasporoporia sensu lato. Megasporia tropica is similar to Megasporoporia inflata by occurring in tropical China, almost the same size of pores, strongly dextrinoid skeletal hyphae, the absence of hyphal pegs and dendrohyphidia, but the former has cystidioles and bigger basidiospores $(14.7-18.8 \times 5-6.5 \mu \mathrm{m}$ vs. $10-11.8 \times 3.5-4.2 \mu \mathrm{m})$, and its skeletal hyphae are unchanged in KOH (Yuan et al. 2017). Phylogenetically, M. inflata is closely related to 
M. bannaensis (Figs 1-2), but the latter species has bigger pores (1-2 per mm) and their skeletal hyphae unchanged in KOH (Li \& Cui 2013a).

Megasporoporiella australiae Y.C. Dai, Yuan Yuan \& Ya.R. Wang, sp. nov.

Figs 9-10

Index Fungorum number: IF558814; Facesoffungi number: FoF10473

Etymology - australiae (Lat.): referring to the species found from Australia.

Basidiocarps annual, resupinate, corky, without odor or taste when fresh, becoming hard corky and cracked upon drying, up to $5.4 \mathrm{~cm}$ long, $2 \mathrm{~cm}$ wide, and $0.4 \mathrm{~mm}$ thick at center; sterile margin distinct, white to cream, cottony, up to $3 \mathrm{~mm}$ wide. Pore surface white to cream when fresh, vinaceous buff to fulvous when dry; pores round to angular, 3-4 per mm; dissepiments thick, entire; subiculum pale buff, corky, up to $0.2 \mathrm{~mm}$ thick; tubes cream, paler than subiculum, corky, up to $0.2 \mathrm{~mm}$ long. Hyphal system dimitic; generative hyphae bearing clamp connections; skeletal hyphae IKI-, $\mathrm{CB}+$; tissues unchanged in $\mathrm{KOH}$ (not dissolved). Subicular generative hyphae infrequent, hyaline, thin-walled, occasionally branched, 2-3 $\mu \mathrm{m}$ in diam; skeletal hyphae dominant, thick-walled with a narrow to medium lumen, frequently branched, strongly flexuous, strongly interwoven, 2-3.5 $\mu \mathrm{m}$ in diam. Tramal generative hyphae hyaline, thin-walled, occasionally branched, 2-2.5 $\mu \mathrm{m}$ in diam; skeletal hyphae dominant, thick-walled with a narrow lumen, moderately branched, strongly flexuous, strongly interwoven, 2-3 $\mu \mathrm{m}$ in diam. Dendrohyphidia absent. Hyphal pegs absent. Cystidia absent; cystidioles present, tubular to fusoid, thin-walled, smooth, 29-41 $\times 2.5-11.5 \mu \mathrm{m}$. Basidia pear-shaped, with four sterigmata and a basal clamp connection, 35-38 $\times$ 9.5-12 $\mu \mathrm{m}$; Basidioles in shape similar to basidia, but smaller, some with a few guttules. All the hymenial cells (cystidioles, basidia and basidioles) with abundant oily substance. Small tetrahedric or polyhedric crystals frequently present among hymenium. Basidiospores cylindrical, hyaline, thin-walled, smooth, sometimes with one big guttule, IKI-, CB-, (11.5-)11.8-15(-16.5) $\times(3.5-) 4-6(-6.5) \mu \mathrm{m}, \mathrm{L}=13.4 \mu \mathrm{m}, \mathrm{W}=4.98 \mu \mathrm{m}, \mathrm{Q}=2.52-2.64$ $(n=60 / 2)$.

Known distribution - widespread in temperate region.

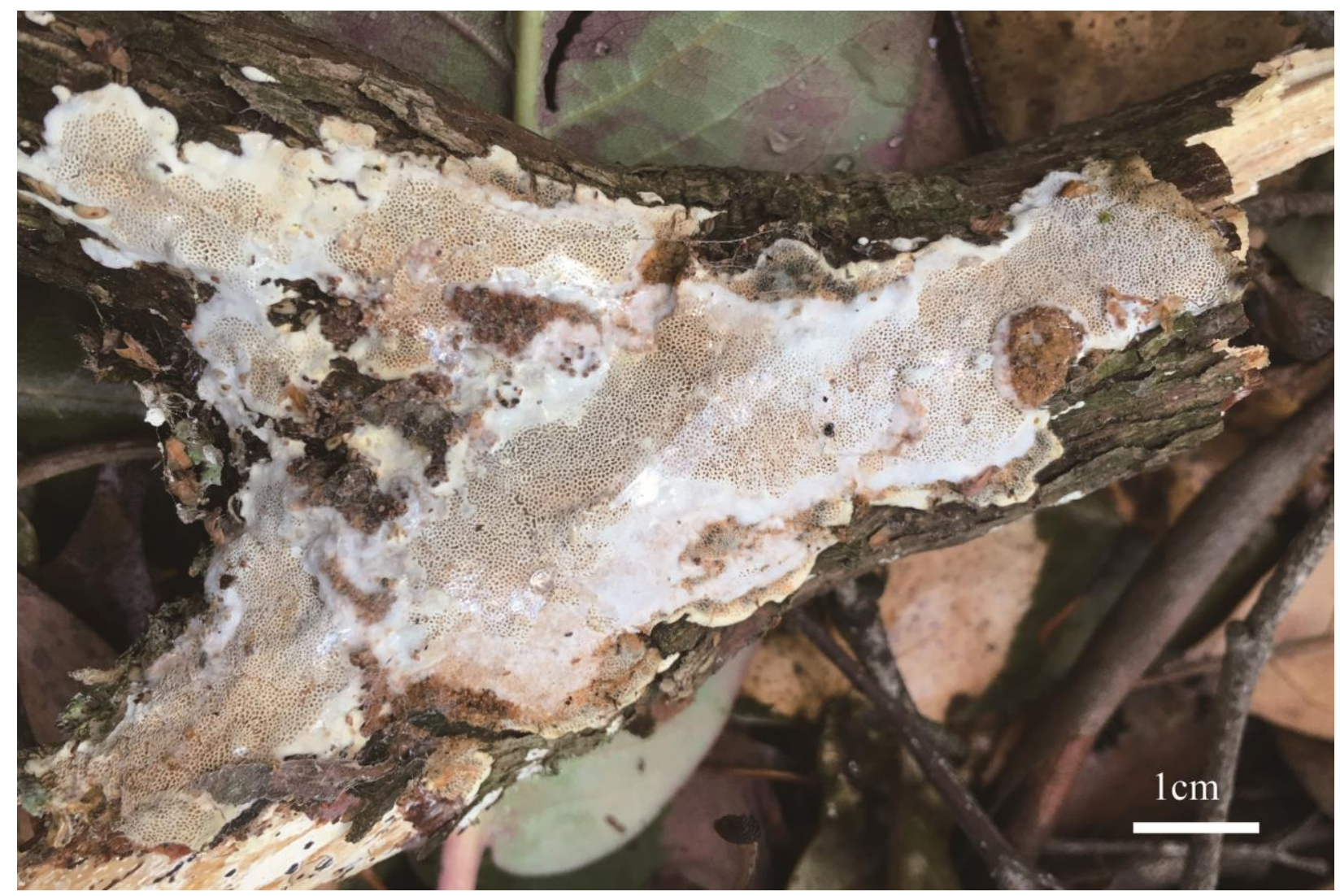

Figure 9 - Basidiocarp of Megasporoporiella australiae (the paratype, Dai 18658). 


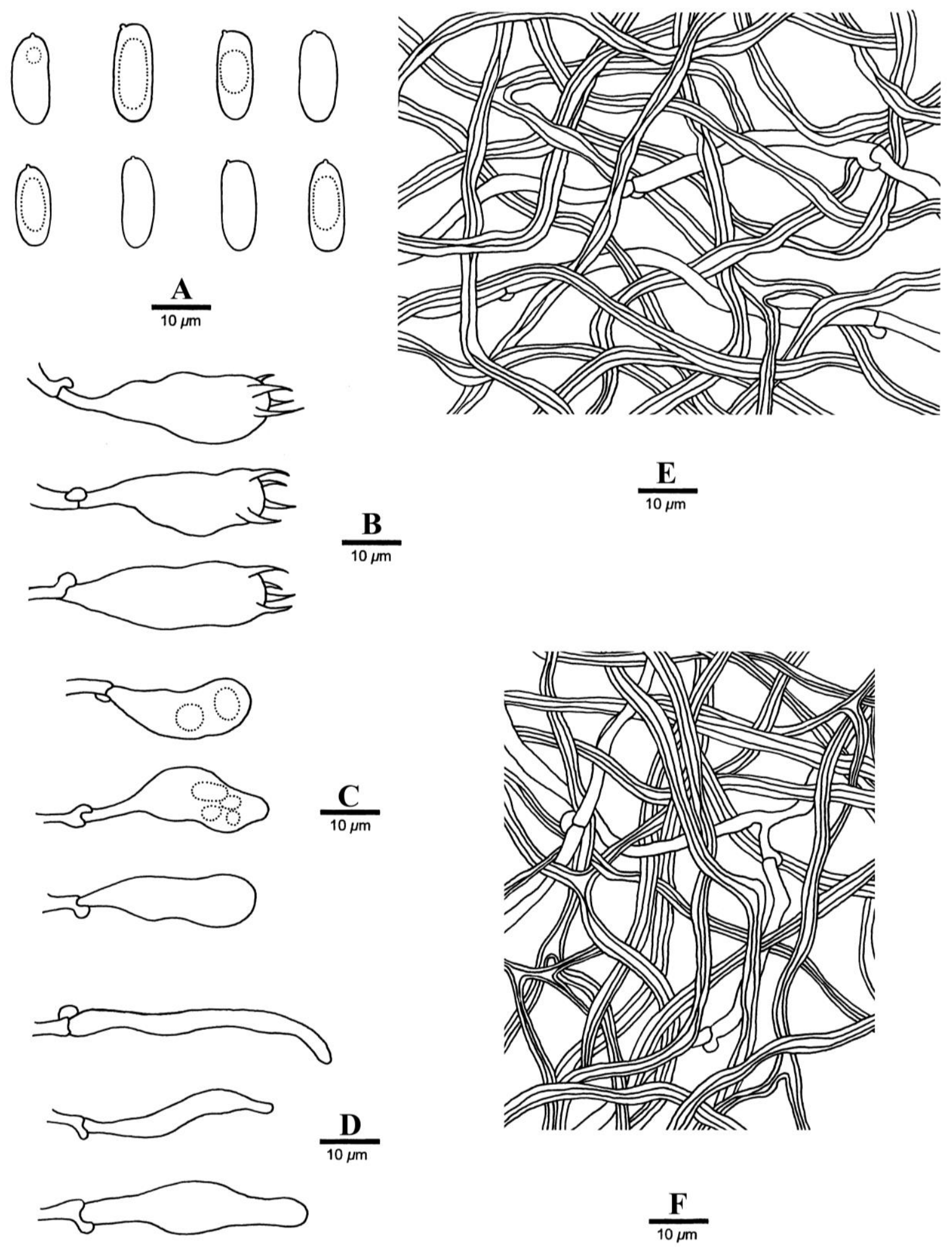

Figure 10 - Microscopic structures of Megasporoporiella australiae (drawn from the holotype, Dai 18658). A Basidiospores. B Basidia. C Basidioles. D Cystidioles. E Hyphae from subiculum. F Hyphae from tubes.

Materials examined - Australia. Melbourne. Dandenong Ranges Botanic Garden, on dead tree of Rhododendron, 12 May 2018, Y.C. Dai 18657 (BJFC27125, holotype), Y.C. Dai 18658 (BJFC27126). 
Notes - Morphologically, Megasporoporiella australiae resembles Megasporia cystidiolophora, Megasporia hengduanensis and Megasporoporia cavernulosa by sharing almost the same size of pores and basidiospores, but the latter three species can be readily distinguished from Megasporoporiella australiae by their dextrinoid skeletal hyphae (Ryvarden et al. 1982, Cui \& Dai 2007, Li \& Cui 2013a). Phylogenetically, M. australiae is closely related to M. rhododendri (Figs 1, 2), but $M$. rhododendri can be distinguished from $M$. australiae by dextrinoid skeletal hyphae and ellipsoid basidiospores measuring as 11-14 × 6.5-8 $\mu \mathrm{m}$ (Dai et al. 2004).

Megasporoporiella hubeiensis (Hai J. Li \& B.K. Cui) Y.C. Dai, Yuan Yuan \& Ya. R. Wang, comb. nov.

Figs 11-12

Index Fungorum number: IF558815; Facesoffungi number: FoF10468

Basidiocarps biennial, resupinate, cushion-shaped, corky, without odor or taste when fresh, becoming hard corky upon drying, up to $2.4 \mathrm{~cm}$ long, $12 \mathrm{~cm}$ wide, and $2.4 \mathrm{~mm}$ thick at center; sterile margin thinning out, very narrow to almost lacking. Pore surface white to cream when fresh, vinaceous buff to fulvous when dry; pores round to angular, 1-2 per mm; dissepiments thick, entire; subiculum pale buff, corky, up to $0.6 \mathrm{~mm}$ thick; tubes concolorous with the pore surface, corky, up to $1.8 \mathrm{~mm}$ long. Hyphal system dimitic; generative hyphae bearing clamp connections; skeletal hyphae IKI-, $\mathrm{CB}+$; tissues unchanged in $\mathrm{KOH}$. (not dissolved). Subicular generative hyphae hyaline, thin-walled, occasionally branched, 2.5-2.8 $\mu \mathrm{m}$ in diam; skeletal hyphae dominant, thick-walled with a narrow to medium lumen, frequently branched, mostly flexuous, interwoven, 2.8-3 $\mu \mathrm{m}$ in diam. Tramal generative hyphae hyaline, thin-walled, occasionally branched, $1.8-2.5$ $\mu \mathrm{m}$ in diam; skeletal hyphae dominant, thick-walled with a narrow lumen, frequently branched, mostly flexuous, interwoven, 2.5-3 $\mu \mathrm{m}$ in diam. Dendrohyphidia present. Hyphal pegs absent. Cystidia absent; cystidioles present, subulate or ventricose, thin-walled, smooth, 18.2-37.2 × 6.3$10.5 \mu \mathrm{m}$. Basidia clavate, with four sterigmata and a basal clamp connection, 26.8-34.2 $\times 7.8-11.2$ $\mu \mathrm{m}$; basidioles in shape similar to basidia, but smaller. Small tetrahedric or polyhedric crystals frequently present among hymenium. Basidiospores cylindrical, hyaline, thin-walled, smooth, usually with one big guttule, IKI-, CB-, (11-)12-14.3(-14.8) $\times(4-) 4.5-5.7(-6.5) \mu \mathrm{m}, \mathrm{L}=13.26$ $\mu \mathrm{m}, \mathrm{W}=5.12 \mu \mathrm{m}, \mathrm{Q}=2.37-2.55(\mathrm{n}=60 / 2)$.

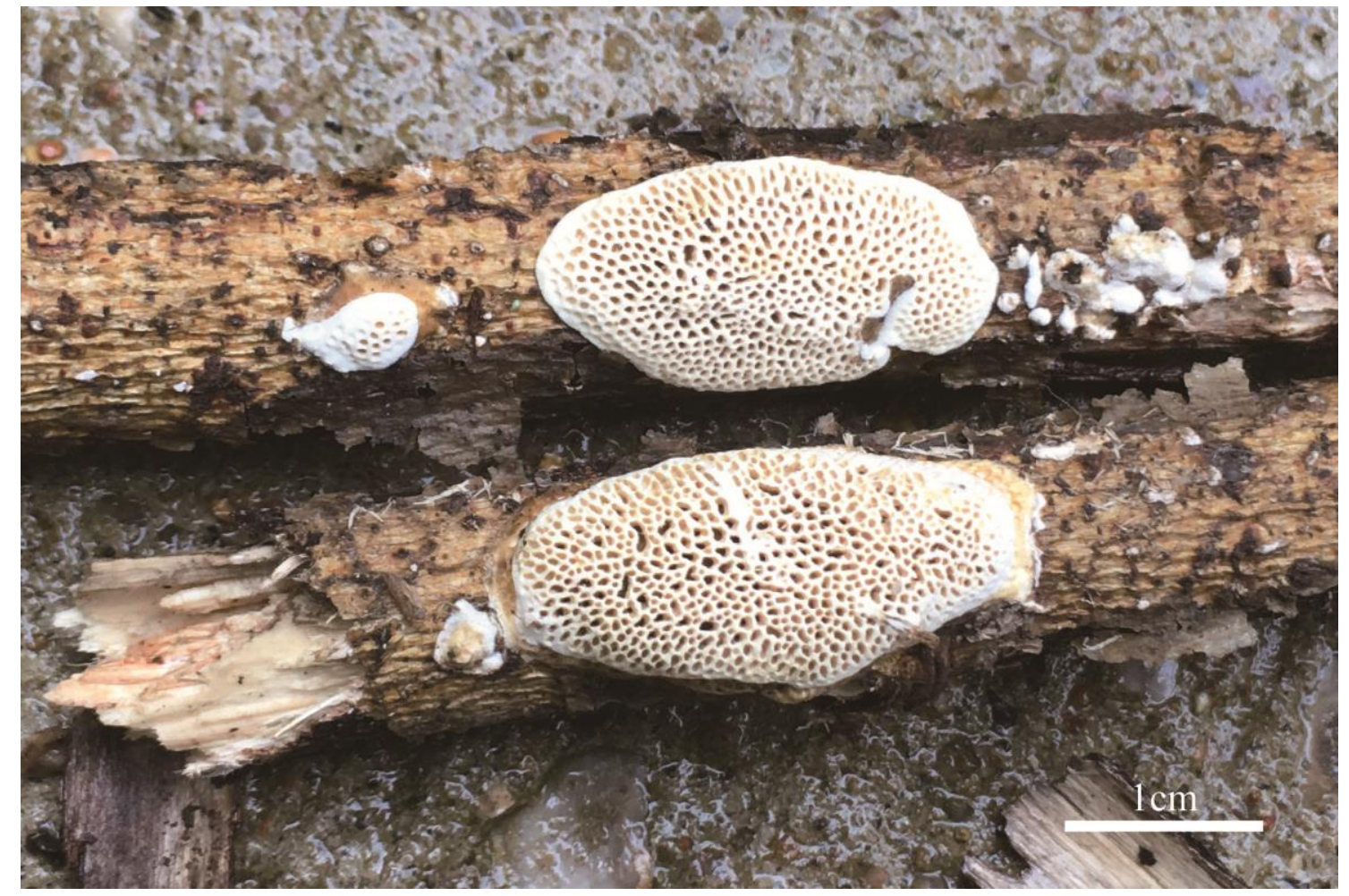

Figure 11 - Basidiocarps of Megasporoporiella hubeiensis (Dai 18102). 

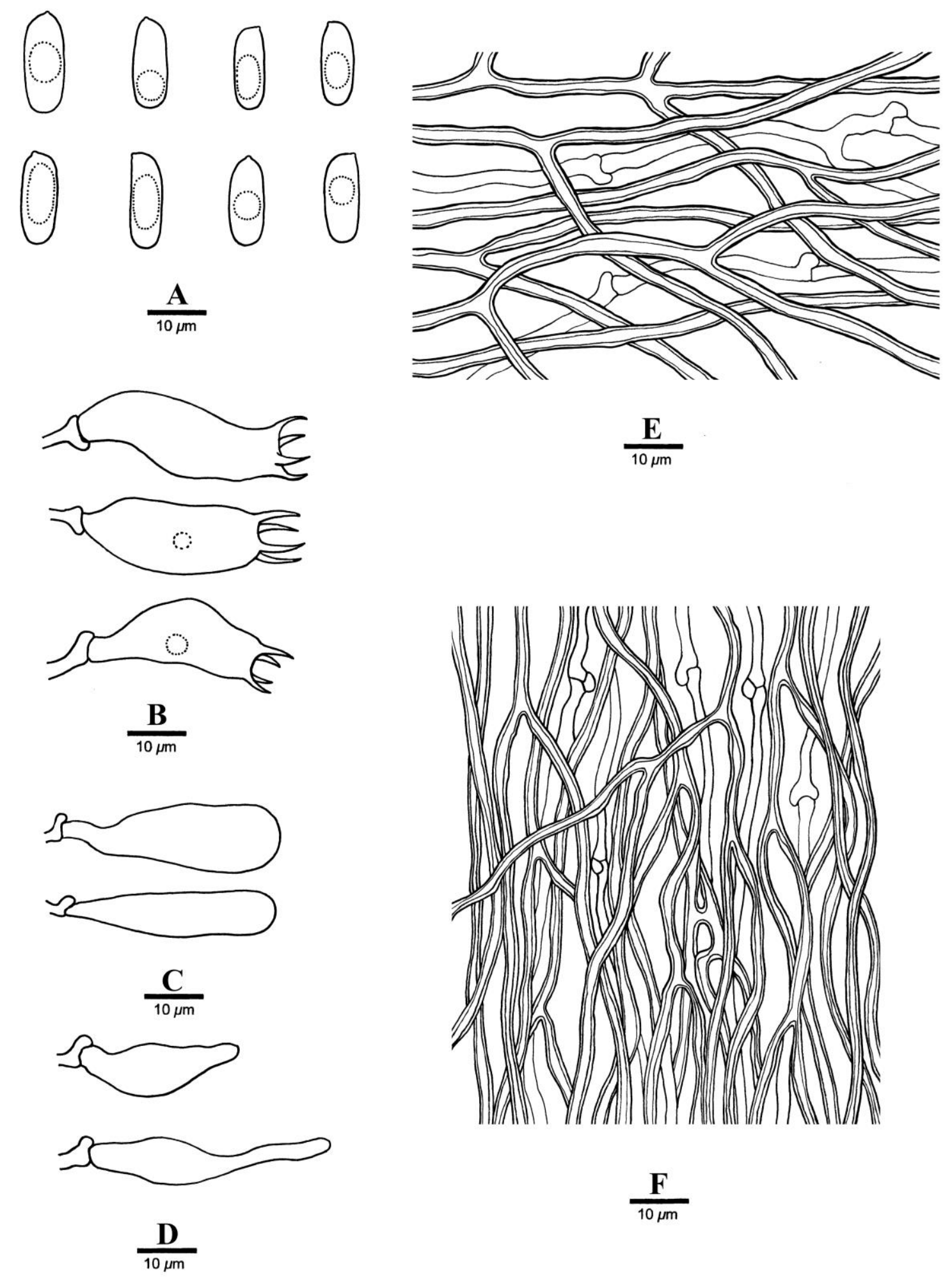

$\frac{\mathbf{E}}{10 \mu \mathrm{m}}$
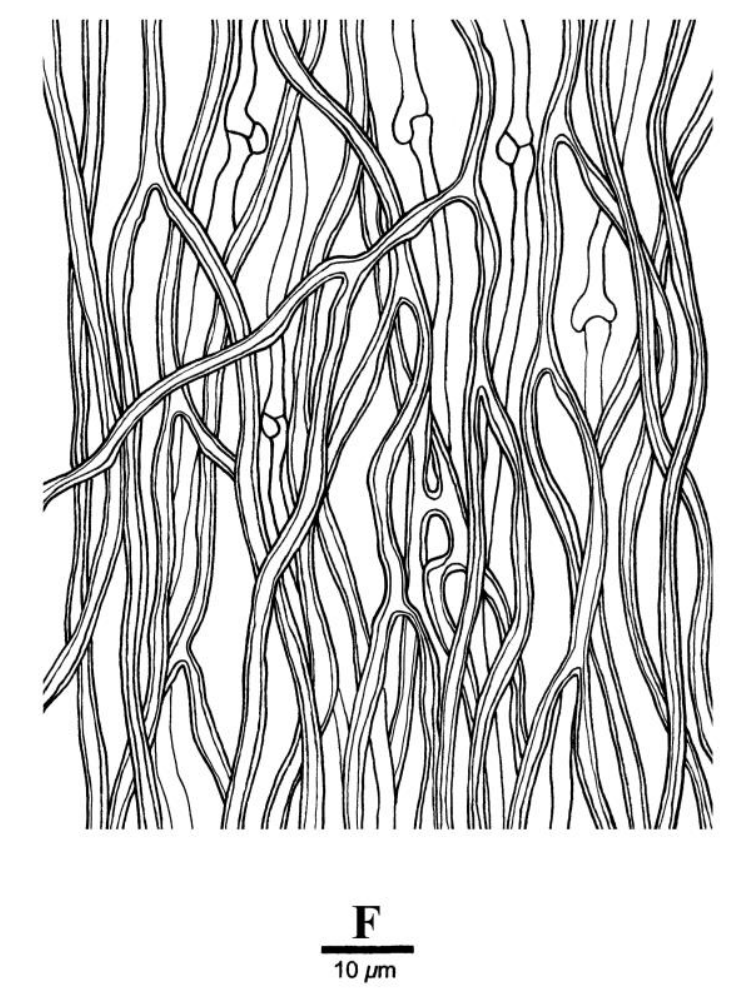

Figure 12 - Microscopic structures of Megasporoporiella hubeiensis (drawn from Dai 18103). A Basidiospores. B Basidia. C Basidioles. D Cystidioles. E Hyphae from subiculum. F Hyphae from tube.

$\equiv$ Dichomitus hubeiensis Hai J. Li \& B.K. Cui, Nordic Journal Botany 31: 118 (2013).

Known distribution - widespread in temperate and subtropical regions.

Materials examined - China, Hebei, Zhuolu County, Xiaowutai Natural Reserve, Shanjiankou, on dead branch of Salix, 10 September 2017, Y.C. Dai 18102 (BJFC025632), Y.C. 
Dai 18103 (BJFC025633); Hubei, Fang County, Shennongjia Natural Reserve, on fallen angiosperm branch, 22 September 2004 , Wei 2045 (BJFC012314, holotype).

Notes - Megasporoporiella hubeiensis was originally described as Dichomitus hubeiensis Hai J. Li \& B.K. Cui from subtropical China (Li \& Cui 2013b). But our phylogenies (Figs 1-2) show the species nested in Megasporoporiella clade with a robust support (100\% MP, 100\% ML, 1.00 BPP). So, the above combination is proposed.

In addition, Li \& Cui (2013a) defined Megasporoporiella cavernulosa as type species of the genus (Li \& Cui 2013a), and they used the specimen $\mathrm{Wu}$ 9508-328 (AY333800) for phylogeny, however, the specimen represents Megasporoporiella subcavernulosa rather than Megasporoporia cavernulosa (Dai \& Wu 2004). Megasporoporia cavernulosa was originally described from America, specimens JV0904/50J, JV0904/52J and JV0904/81 collected from America are analyzed, and they are distantly related to Megasporoporiella (Fig. 1). In the present study we select Megasporoporiella pseudocavernulosa as the type species of Megasporoporiella.

For conveniences for the readers, a comparison of main characteristics (Table 3) and an identification key of Megasporia, Megasporoporia sensu stricto and Megasporoporiella are provided as following.

Table 3 The main characteristics of species of Megasporia, Megasporoporia sensu stricto and Megasporoporiella

\begin{tabular}{|c|c|c|c|c|c|c|c|c|c|c|c|}
\hline Species & $\begin{array}{l}\text { Pores } \\
\text { (per mm) }\end{array}$ & $\begin{array}{l}\text { Dextrinoid } \\
\text { of skeletals }\end{array}$ & $\begin{array}{l}\text { KOH } \\
\text { reaction of } \\
\text { skeletals }\end{array}$ & Cystidioles & Gloeocystidioles & Basidia $(\mu \mathrm{m})$ & $\begin{array}{l}\text { Shape of } \\
\text { basidiospores }\end{array}$ & $\begin{array}{l}\text { Basidiospores } \\
(\mu \mathrm{m})\end{array}$ & Dendrohyphidia & $\begin{array}{l}\text { Hyphal } \\
\text { pegs }\end{array}$ & References \\
\hline Megasporia bambusae & $4-5$ & {$[+]$} & {$[-]$} & + & - & $\begin{array}{l}20-35 \times 10- \\
15\end{array}$ & ellipsoid & $\begin{array}{l}11.8-14 \times 5.8- \\
6.8\end{array}$ & + & - & $\begin{array}{l}\text { Present } \\
\text { study }\end{array}$ \\
\hline M. cystidiolophora & $3-5$ & {$[++]$} & {$[-]$} & + & - & $18-25 \times 7-10$ & cylindrical & $\begin{array}{l}11.7-14.9 \times \\
4.1-5.6\end{array}$ & - & - & $\begin{array}{l}\text { Cui \& Dai } \\
\text { (2007) }\end{array}$ \\
\hline M. ellipsoidea & $1-1.5$ & {$[+]$} & {$[-]$} & + & + & $23-40 \times 9-15$ & ellipsoid & $12-15 \times 6-8.2$ & + & + & $\begin{array}{l}\text { Du \& Cui } \\
(2009)\end{array}$ \\
\hline M. fusiformis & $3.5-4$ & {$[-]$} & {$[-]$} & + & - & $25-38 \times 8-12$ & fusiform & $\begin{array}{l}15-19.8 \times 4.2- \\
6.8\end{array}$ & + & - & $\begin{array}{l}\text { Present } \\
\text { study }\end{array}$ \\
\hline M. guangdongensis & $4-5$ & {$[+++]$} & {$[-]$} & + & - & $20-28 \times 5-8$ & cylindrical & $\begin{array}{l}11-14.9 \times 3.4- \\
4.5\end{array}$ & - & - & $\begin{array}{l}\text { Li \& Cui } \\
\text { (2013a) }\end{array}$ \\
\hline M. hengduanensis & $2-3$ & {$[++]$} & {$[-]$} & + & - & $30-37 \times 9-12$ & cylindrical & $\begin{array}{l}11-15 \times 4.2- \\
5.2\end{array}$ & - & - & $\begin{array}{l}\text { Li \& Cui } \\
\text { (2013a) }\end{array}$ \\
\hline M. hexagonoides & $0.5-1$ & {$[+]$} & {$[-]$} & + & - & $38-40 \times 8-12$ & allantoid & $17-21 \times 5-6$ & - & - & $\begin{array}{l}\text { Dai \& Cui } \\
\text { (2008) }\end{array}$ \\
\hline M. major & $1-1.5$ & {$[+++]$} & {$[+]$} & + & - & $\begin{array}{l}24-38 \times 12- \\
16\end{array}$ & cylindrical & $\begin{array}{l}15.2-20 \times 5.5- \\
7.1\end{array}$ & + & + & $\begin{array}{l}\text { Dai \& Li } \\
\text { (2002) }\end{array}$ \\
\hline M. rimosa & $3-4$ & {$[+]$} & {$[-]$} & + & - & $20-28 \times 5-8$ & cylindrical & $\begin{array}{l}16.8-20.2 \times \\
4.3-5.5\end{array}$ & + & - & $\begin{array}{l}\text { Yuan et al. } \\
\text { (2017) }\end{array}$ \\
\hline M. tropica & $2-3$ & {$[+++]$} & {$[-]$} & + & - & $20-25 \times 7-10$ & cylindrical & $\begin{array}{l}14.7-18.8 \times 5- \\
6.5\end{array}$ & - & - & $\begin{array}{l}\text { Yuan et al. } \\
\text { (2017) }\end{array}$ \\
\hline
\end{tabular}


Table 3 Continued.

\begin{tabular}{|c|c|c|c|c|c|c|c|c|c|c|c|}
\hline Species & $\begin{array}{l}\text { Pores } \\
\text { (per mm) }\end{array}$ & $\begin{array}{l}\text { Dextrinoid } \\
\text { of skeletals }\end{array}$ & $\begin{array}{l}\mathrm{KOH} \\
\text { reaction of } \\
\text { skeletals }\end{array}$ & Cystidioles & Gloeocystidioles & Basidia $(\mu \mathrm{m})$ & $\begin{array}{l}\text { Shape of } \\
\text { basidiospores }\end{array}$ & $\begin{array}{l}\text { Basidiospores } \\
(\mu \mathrm{m})\end{array}$ & Dendrohyphidia & $\begin{array}{l}\text { Hyphal } \\
\text { pegs }\end{array}$ & References \\
\hline M. violacea & $5-7$ & {$[+++]$} & {$[-]$} & + & - & $13-19 \times 5-10$ & cylindrical & $\begin{array}{l}11-14.9 \times 3.2- \\
5\end{array}$ & + & - & $\begin{array}{l}\text { Du \& Cui } \\
\text { (2009) }\end{array}$ \\
\hline M. yunnanensis & $2-3$ & {$[+]$} & {$[-]$} & + & - & $30-35 \times 9-11$ & cylindrical & $\begin{array}{l}15-20.8 \times 5.5- \\
7.1\end{array}$ & + & - & $\begin{array}{l}\text { Yuan et al. } \\
\text { (2017) }\end{array}$ \\
\hline $\begin{array}{l}\text { Megasporoporia } \\
\text { bannaensis }\end{array}$ & $1-2$ & {$[+++]$} & {$[-]$} & + & - & $20-32 \times 8-10$ & cylindrical & $\begin{array}{l}10-14 \times 3.9- \\
4.6\end{array}$ & - & + & $\begin{array}{l}\text { Li \& Cui } \\
\text { (2013a) }\end{array}$ \\
\hline M. inflata & $2.5-3$ & {$[+++]$} & {$[+++]$} & - & - & $14-23 \times 7-9$ & cylindrical & $\begin{array}{l}10-11.8 \times 3.5- \\
4.2\end{array}$ & - & - & $\begin{array}{l}\text { Present } \\
\text { study }\end{array}$ \\
\hline M. minor & $6-7$ & {$[+++]$} & {$[-]$} & + & - & $18-26 \times 6-8$ & ellipsoid & $6-7.8 \times 2.6-4$ & - & + & $\begin{array}{l}\text { Li \& Cui } \\
\text { (2013a) }\end{array}$ \\
\hline M. setulosa & $1-2$ & {$[+++]$} & {$[-]$} & - & - & $18-29 \times 7-10$ & cylindrical & $\begin{array}{l}10-14 \times 4.2- \\
5.7\end{array}$ & - & + & $\begin{array}{l}\text { Ryvarden } \\
\text { et al. } \\
(1982)\end{array}$ \\
\hline $\begin{array}{l}\text { Megasporoporiella } \\
\text { australiae }\end{array}$ & $3-4$ & {$[-]$} & {$[-]$} & + & + & $\begin{array}{l}35-38 \times 10- \\
12\end{array}$ & cylindrical & $11.8-15 \times 4-6$ & - & - & $\begin{array}{l}\text { Present } \\
\text { study }\end{array}$ \\
\hline M. hubeiensis & $1-2$ & {$[-]$} & {$[-]$} & + & - & $27-34 \times 8-11$ & cylindrical & $\begin{array}{l}12-14.3 \times 4.5- \\
5.7\end{array}$ & + & - & $\begin{array}{l}\text { Li \& Cui } \\
\text { (2013a), } \\
\text { Present } \\
\text { study }\end{array}$ \\
\hline M. pseudocavernulosa & $1.5-2.5$ & {$[++]$} & {$[-]$} & + & - & $\begin{array}{l}34-52 \times 10- \\
12\end{array}$ & allantoid & $\begin{array}{l}10.8-14 \times 5.3- \\
6.5\end{array}$ & - & + & $\begin{array}{l}\mathrm{Li} \& \mathrm{Cui} \\
(2013 \mathrm{a})\end{array}$ \\
\hline M. rhododendri & $4-5$ & {$[++]$} & {$[-]$} & + & - & $23-40 \times 9-14$ & ellipsoid & $11-14 \times 6.5-8$ & - & - & $\begin{array}{l}\text { Dai et al. } \\
(2004)\end{array}$ \\
\hline M. subcavernulosa & $2-4$ & {$[+]$} & {$[-]$} & - & - & $18-24 \times 8-11$ & cylindrical & $\begin{array}{l}9-12.1 \times 4.2- \\
5.2\end{array}$ & + & + & $\begin{array}{l}\text { Dai \& Wu } \\
\text { (2004) }\end{array}$ \\
\hline
\end{tabular}

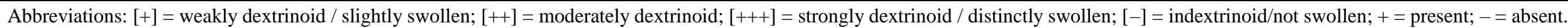

Key to known species of Megasporia, Megasporoporia sensu stricto and Megasporoporiella

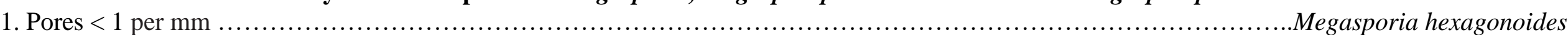

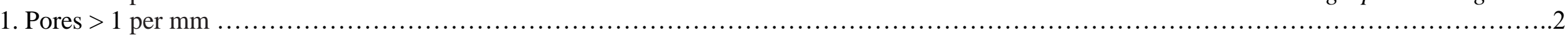

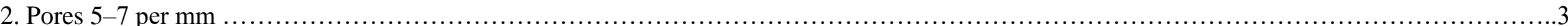

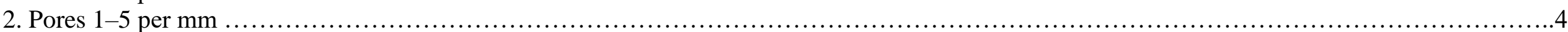

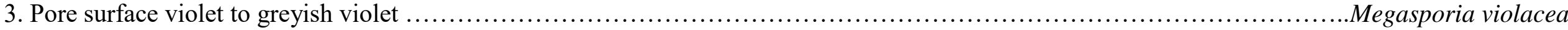

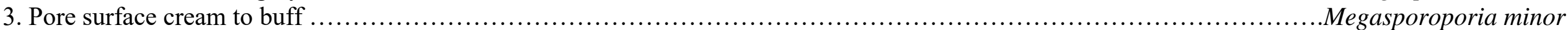

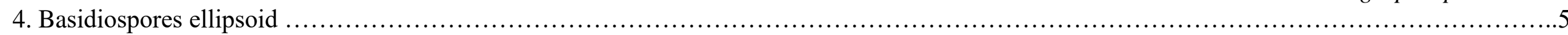


4. Basidiospores cylindrical, allantoid or fusiform

5. Pores $1-2$ per $\mathrm{mm}$

5. Pores $4-5$ per mm Megasporia ellipsoidea

6. Dendrohyphidia present; on bamboo Megasporia bambusae

6. Dendrohyphidia absent; on Rhododendron oroporiella rhododendri

7. Basidiospores fusiform Megasporia fusiformis

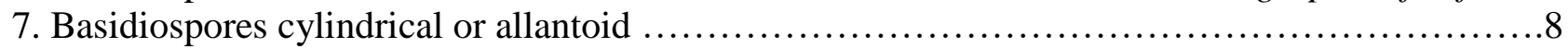

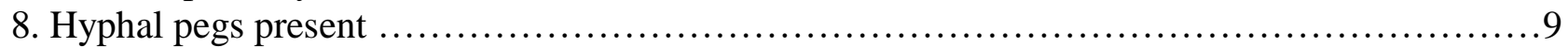

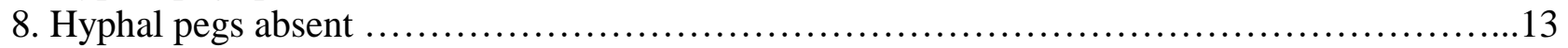

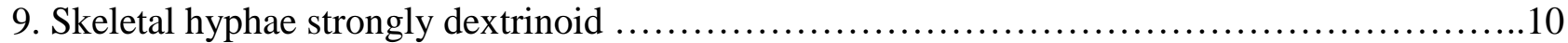

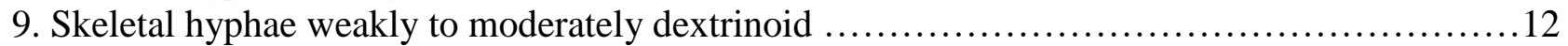

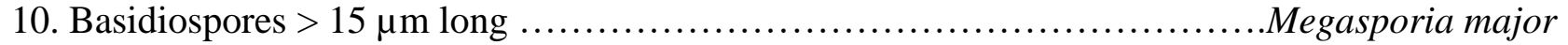

10. Basidiospores $<15 \mu \mathrm{m}$ long ....................................................... 11

11. Cystidioles present; Asian species

Megasporoporia bannaensis

11. Cystidioles absent; African and American species Megasporoporia setulosa

12. Dendrohyphidia present, cystidioles absent Megasporoporiella subcavernulosa

12. Dendrohyphidia absent, cystidioles present Megasporoporiella pseudocavernulosa

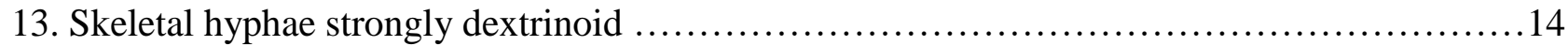

13. Skeletal hyphae indextrinoid, weakly to moderately dextrinoid ........................... 16

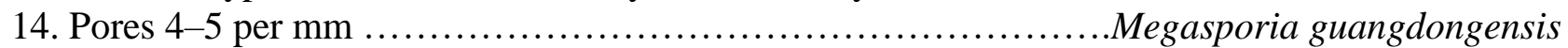

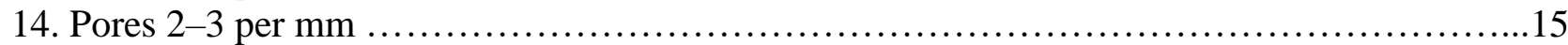

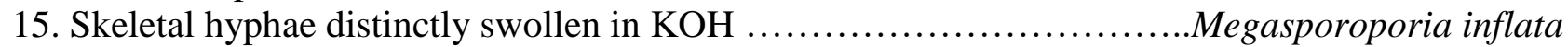

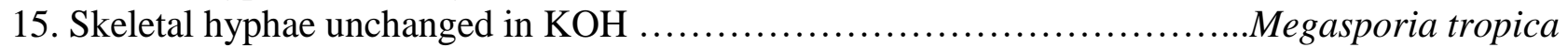

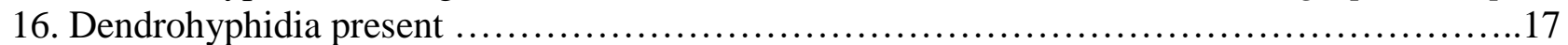

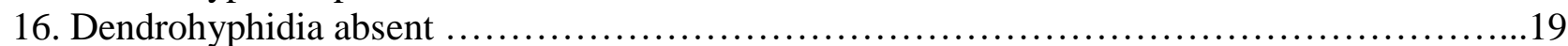

17. Basidiospores < $15 \mu \mathrm{m}$ long ..................................egasporoporiella hubeiensis

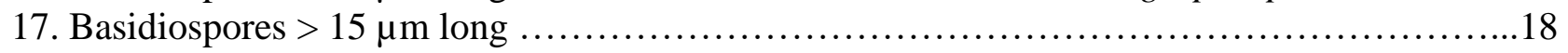

18. Basidiocarps cracked when dry ....................................... gasporia rimosa

18. Basidiocarps uncracked when dry ..................................egasporia yunnanensis

19. Gloeocystidioles present, skeletal hyphae indextrinoid .............Megasporoporiella australiae

19. Gloeocystidioles absent, skeletal hyphae moderately dextrinoid ..........................20

20. Pore surface cream to buff, pores $2-3$ per $\mathrm{mm}$......................Megasporia hengduanensis

20. Pore surface pale pinkish brown to salmon, pores 3-5 per mm ......Megasporia cystidiolophora

\section{Discussion}

Dichomitus D.A. Reid resembles Megasporoporia sensu lato by light colored and resupinate basidiocarps, a dimitic hyphal system with generative hyphae bearing clamp connections, cyanophilous skeletal hyphae, and hyaline, thin-walled basidiospores; that is why Masuka \& Ryvarden (1999), Robledo \& Rajchenberg (2007) merged them, but without molecular analysis. Type species of Dichomitus, D. squalens, is included in our phylogeny and it is distantly related to Megasporia, Megasporoporia sensu stricto, Megasporoporiella, and the unnamed clade of Megasporoporia sensu lato. (Figs 1-2). Morphologically, Dichomitus lacks hyphal pegs and dendrohyphidia, its skeletal hyphae are indextrinoid and dendritically branched in which they are very similar to Polyporus sensu stricto; while the most species of Megasporia, Megasporoporia sensu stricto and Megasporoporiella have hyphal pegs and dendrohyphidia, and their skeletal hyphae are dextrinoid in most species. So, we treat Dichomitus, Megasporia, Megasporoporia sensu stricto and Megasporoporiella as independent genera.

As mentioned in the introduction, Megasporia, Megasporoporia sensu stricto and Megasporoporiella are distinct in phylogeny, but it is impossible to separate each other by morphology. So, we treat our new species and the combination in Megasporia, Megasporoporia sensu stricto and Megasporoporiella, but for the discussion of their similar species, all the members of Megasporoporia sensu lato are included. In our phylogeny (Figs 1, 2), Dichomitus amazonicus, 
Megasporoporia cavernulosa and M. mexicana are nested in three lineages and formed a clade. Dichomitus amazonicus was described from neotropics (Gomes-Silva et al. 2012), corresponding specimen URM 87859 is examined, and the specimen has strongly dextrinoid skeletal hyphae and hyphal pegs (not mentioned in the original description). Megasporoporia cavernulosa was originally described from Brazil (Ryvarden 1984), and it has dendrohyphidia and dextrinoid skeletal hyphae. Megasporoporia mexicana was originally described from Mexico (Ryvarden et al. 1982), and it was combined in Dichomitus without DNA data (Ryvarden 2007). A specimen of Megasporoporia mexicana from Honduras (JV1806/4J, sequenced) is studied, and it has hyphal pegs and dextrinoid skeletal hyphae. So, all these three species have dextrinoid skeletal hyphae which are different from $D$. squalens (the type species of Dichomitus). Phylogenetically, these three species are distantly related to Dichomitus, Megasporia, Megasporoporia sensu stricto and Megasporoporiella. A new genus might be set up to accommodate them, for the time being, we treat them as present names because more materials need to be examined and phylogenetically analyzed.

Megasporoporia minuta Y.C. Dai \& X.S. Zhou was described from China without phylogenetic analysis (Zhou \& Dai 2008), but our phylogenies show its type is distantly related to Megasporoporia sensu lato (Figs 1-2). Its type (Zhou 120) and another specimen Cui 13945 are studied, they have perennial basidiocarps, small pores (6-8 per $\mathrm{mm})$, indextrinoid skeletal hyphae, narrowly ovoid basidiospores, lack hyphal pegs, dendrohyphidia and tetrahedric or polyhedric crystals. These characteristics do not fit the definition of Megasporoporia sensu lato. So, the species is excluded from Megasporia, Megasporoporia sensu stricto and Megasporoporiella. Moreover, Megasporoporiella lacerata B.K. Cui \& Hai J. Li was described from China based on morphology and a single gene phylogeny (Li \& Cui 2013a). Although its morphological characteristics are similar to that of Megasporoporia sensu lato. However, our phylogenies based on 2-gene and 4-gene sequences datasets show that M. lacerata is closer to Polyporus tuberaster (type species of Polyporus sensu stricto, Figs 1, 2). So, the species should be combined into Polyporus. However, Polyporus laceratus Berk. (Ann. nat. Hist., Mag. Zool. Bot. Geol. 3: 392, 1839 ) is existed, and the species is re-named as following.

Polyporus megasporoporus Y.C. Dai, Yuan Yuan \& Ya.R. Wang, nomen. nov.

Index Fungorum number: IF558816; Facesoffungi number: FoF10469

Etymology - megasporoporus (Lat.): referring to the species similar with Megasporopoia.

As given by Li and Cui 2013: 377.

= Megasporoporiella lacerata B.K. Cui \& Hai J. Li, Mycologia 105(2): 377 (2013).

Material examined - China. Yunnan Province. Baoshan County, Gaoligong Mountains, Baihualing Nature Reserve, on fallen angiosperm branch, 09 Sep 2007, Yuan 3880 (holotype in IFP, isotype in BJFC).

Besides the species in our phylogeny and the above discussion, the taxa Dichomitus affixus (Corner) T. Hatt. (Hattori 2002), D. africanus Ryvarden (Ryvarden 2019), D. amygdalinus (Berk \& Ravenel) Ryvarden (Ryvarden 1977), D. anoectoporus (Berk \& M.A. Curtis) Ryvarden (Ryvarden 1984), D. cameroonensis Ryvarden (Ryvarden 2018), D. citricremeus Masuka \& Ryvarden (Masuka \& Ryvarden 1999), D. costaricensis Ryvarden (Ryvarden 2012), D. cylindrosporus Ryvarden (Ryvarden 2007), D. delicatulus (Henn.) Masuka \& Ryvarden (Masuka \& Ryvarden 1999), D. densiporus Ryvarden (Ryvarden 2019), D. deviatus Ipulet \& Ryvarden (Ipulet \& Ryvarden 2005), D. ecuadorensis Ryvarden (Læssøe \& Ryvarden 2010), D. efibulatus A.M. Ainsw. \& Ryvarden (Ainsworth \& Ryvarden 2008), D. epitephrus (Berk.) Ryvarden (Cunningham 1965), D. grandisporus Aime \& Ryvarden (Aime et al. 2007), D. leucoplacus (Berk.) Ryvarden (Ryvarden 1977), D. newhookii P.K. Buchanan \& Ryvarden (Buchanan \& Ryvarden 2000), D. papuanus Quanten (Quanten 1996), D. perennis Ryvarden (Ryvarden 2007) \& D. sinuolatus H.S. Yuan (Yuan 2013) are recorded in Dichomitus, but most of these taxa lack DNA data, and we did not yet exam their vouchers, and for the time being they are not included in Megasporoporia 
sensu lato. By comparing the original descriptions of these species, the new species we have recently described are different from them.

\section{Acknowledgements}

We thank Prof. Leif Ryvarden (Norway), Prof. Bao-Kai Cui (China) and Dr. Yu-Lian Wei (China) for allowing us to study their collections. The research is supported by National Natural Science Foundation of China (Project No. 32000010, 31530002).

\section{References}

Aime L, Ryvarden L, Henkel TW. 2007 - Studies in neotropical polypores 22. Additional new and rare species from Guyana. Synopsis Fungorum 23, 15-31.

Ainsworth AM, Ryvarden L. 2008 - Dichomitus efibulatus species nova. Synopsis Fungorum 25, $48-52$.

Anonymous. 1969 - Flora of British fungi. Color identification chart. Her Majesty's Stationery Office, London.

Buchanan PK, Ryvarden L. 2000 - New Zealand polypore fungi: six new species and a redetermination. New Zealand Journal of Botany 38, 251-263.

Doi 10.1080/0028825X.2000.9512682

Chen JJ, Cui BK, Dai YC. 2016 - Global diversity and phylogeny of Wrightoporia (Russulales, Basidiomycota). Persoonia 37, 21-36. Doi 10.3767/003158516X689666

Cui BK, Dai YC. 2007 - Polypores from Tianmushan Nature Reserve (Zhejiang Province, eastern China). Mikologiya i Fitopatologiya 41, 506-514.

Cui BK, Li HJ, Ji X, Zhou JL et al. 2019 - Species diversity, taxonomy and phylogeny of Polyporaceae (Basidiomycota) in China. Fungal Diversity 97, 137-392.

Doi 10.1007/s13225-019-00427-4

Cunningham GH. 1965 - Polyporaceae of New Zealand. - NZ Dep. Sci. Ind. Res. Bull. 164, 1304.

Dai YC, Cui BK. 2008 - Notes on Megasporoporia (Basidiomycota, Polyporales) in China. Mycosystema 27, 604-607.

Dai YC, Li TH. 2002 - Megasporoporia major (Basidiomycota), a new combination. Mycosystema 4, 519-521.

Dai YC, Wei YL, Wang Z. 2004 - Wood-inhabiting fungi in southern China 2. Polypores from Sichuan Province. Ann. Bot. Fenn. 41, 319-329.

Dai YC, Wu SH. 2004 - Megasporoporia (Aphyllophorales, Basidiomycota) in China. Mycotaxon 89, 379-388.

Du P, Cui BK. 2009 - Two new species of Megasporoporia (Polyporales, Basidiomycota) from tropical China. Mycotaxon 110, 131-138. Doi 10.5248/110.131

Farris JS. 1989 - The retention index and the rescaled consistency index. Cladistics 5, 417-419. Doi 10.1111/j.1096-0031. 1989. tb00573.x

Farris JS, Källersjö M, Kluge AG, Bult C. 1994 - Testing significance of incongruence. Cladistics 10, 315-319. Doi 10.1111/j.1096-0031.1994.tb00181.x

Felsenstein J. 1985 - Confidence intervals on phylogenetics: an approach using bootstrap. Evolution 39, 783-791. Doi 10.2307/2408678

Gomes-Silva AC, Ryvarden L, Gibertoni TB. 2012 - Resupinate poroid fungi from tropical rain forests in Brazil: two new species and new records. Mycological Progress 11, 879-885. Doi 10.1007/s11557-011-0803-9

Hall TA. 1999 - Bioedit: a user-friendly biological sequence alignment editor and analysis program for Windows 95/98/NT. Nucleic Acids Symp. Ser. 41, 95-98.

Hattori T. 2002 - Type studies of the polypores described by E.J.H. Corner from Asia and the West Pacific IV. Species described in Tyromyces (1). Mycoscience 43, 307-315. Doi 10.1007/s102670200045 
Ipulet P, Ryvarden L. 2005 - New and interesting polypores from Uganda. Synopsis Fungorum 20, 87-99.

Læssøe T, Ryvarden L. 2010 - Studies in neotropical polypores 26. Some new and rarely recorded polypores from Ecuador. Synopsis Fungorum 27, 34-58.

Li HJ, Cui BK. 2013a - Taxonomy and phylogeny of the genus Megasporoporia and its related genera. Mycologia 105, 368-383. doi: 10.3852/12-114.

Li HJ, Cui BK. 2013b - Dichomitus hubeiensis sp. nov. and a new record of Dichomitus (Basidiomycota) from China. Nordic Journal of Botany 31, 118-121.

Doi 10.1111/j.1756-1051.2012.01498.x

Li HJ, Cui BK, Dai YC. 2014 - Taxonomy and multi-gene phylogeny of Datronia (Polyporales, Basidiomycota). Persoonia 32, 170-182. Doi 10.3767/003158514X681828

Li HJ, Si J, He SH. 2016 - Daedaleopsis hainanensis sp. nov. (Polyporaceae, Basidiomycota) from tropical China based on morphological and molecular evidence. Phytotaxa 275, 294300.

Masuka A, Ryvarden L. 1999 - Dichomitus in Africa. Mycological Research 103, 1126-1130. Doi 10.1017/S0953756299008436

Maddison WP, Maddison DR. 2009 - Mesquite: a modular system for evolutionary analysis. Evolution 11.

Nylander JAA. 2004 - MrModeltest v2.2 Program distributed by the author. Evolutionary Biology Centre, Uppsala University.

Petersen JH. 1996 - Farvekort. The Danish Mycological Society's color-chart. Greve: Foreningen til Svampekundskabens Fremme, Greve, 1-6 pp.

Posada D, Crandall KA. 1998 - Modeltest: testing the model of DNA substitution. Bioinformatics 14, 817-818. Doi 10.1093/ bioinformatics/14.9.817

Quanten E. 1996 - Polypores from Papua New Guinea: some new species. Mycotaxon 59, 427439.

Rehner SA, Buckley E. 2005 - A Beauveria phylogeny inferred from nuclear ITS and EF1- $\alpha$ sequences: evidence for cryptic diversification and links to Cordyceps teleomorphs. Mycologia 97, 84-98. Doi 10.1080/15572536.2006.11832842

Robledo GL, Rajchenberg M. 2007 - South American polypores: first annotated checklist from Argentinean Yungas. Mycotax on 100, 5-9.

Ronquist F, Huelsenbeck JP. 2003 - MrBayes 3: bayesian phylogenetic inference under mixed models. Bioinformatics 19, 1572-1574. Doi 10.1093/bioinformatics/btg180

Ryvarden L. 1977 - Type-studies in the Polyporaceae 10. Species described by J.M. Berkeley, either alone or with other authors from 1844 to 1855. Norwegian Journal of Botany 24, 213230.

Ryvarden L. 1984 - Type studies in the Polyporaceae 16. Species described by J.M. Berkeley, either alone or with other mycologists from 1856 to 1886. Mycotaxon 20, 329-363.

Ryvarden L. 2007 - Studies in neotropical polypores 23. New and interesting wood-inhabiting fungi from Belize. Synopsis Fungorum 23, 32-55.

Ryvarden L. 2012 - Studies in neotropical polypores 32. Synopsis Fungorum 30, 33-43.

Ryvarden L. 2018 - Studies in African Aphyllophorales 25. New poroid species from East and Central Africa. Synopsis Fungorum 38, 25-32.

Ryvarden L. 2019 - Studies in African Aphyllophorales 32. Some new African polypores. Synopsis Fungorum 39, 59-71.

Ryvarden L, Wright JE, Rajchenberg M. 1982 - Megasporoporia, a new genus of resupinate polypores. Mycotaxon 16, 172-182.

Shen LL, Wang M, Zhou JL, Xing JH et al. 2019 - Taxonomy and phylogeny of Postia. Multigene phylogeny and taxonomy of the brown-rot fungi: Postia (Polyporales, Basidiomycota) and related genera. Persoonia 42, 101-126. Doi 10.3767/persoonia.2019.42.05

Stamatakis A. 2014 - RAxML Version 8: a tool for phylogenetic analyses and post analyses of large phylogenies. Bioinformatics 30, 1312-1313. Doi 10.1093/bioinformatics/btu033 
Sun YF, Costa-Rezende DH, Xing JH, Zhou JL et al. 2020 - Multi-gene phylogeny and taxonomy of Amauroderma s. lat. (Ganodermataceae). Persoonia 44, 206-239.

Doi 10.3767/persoonia.2020.44.08

Swofford DL. 2002 - PAUP*: Phylogenetic analysis using parsimony (*and other methods), version 4.0 Beta. Sunderland, MA: Sinauer.

Thompson JD, Gibson TJ, Plewniak F, Jeanmougin F, Higgins DG. 1997 - The CLUSTAL X windows interface: flexible strategies for multiple sequence alignment aided by quality analysis tools. Nucleic Acids Research 25, 4876-4882. Doi 10.1093/nar/25.24.4876

Vilgalys R, Hester M. 1990 - Rapid genetic identification and mapping of enzymatically amplified ribosomal DNA from several Cryptococcus species. Journal of Bacteriology 172, 42384246. Doi 10.1128/jb.172.8.4238-4246.1990

White TJ, Bruns T, Lee S, Taylor JW. 1990 - Amplification and direct sequencing of fungal ribosomal RNA genes for phylogenetics. pp. 315-322 in: Innis, M.A., Gelfand, D.H., Sninsky, J.J. and White, T.J. (eds.), PCR protocols: a guide to methods and applications. New York: Academic Press. Doi 10.1016/B978-0-12-372180-8.50042-1

Yuan HS. 2013 - Dichomitus sinuolatus sp. nov. (Basidiomycota, Polyporales) from China and a key to the genus. Nova Hedwigia 97, 495-501. Doi 10.1127/0029-5035/2013/0098

Yuan Y, Ji XH, Chen JJ, Dai YC. 2017 - Three new species of Megasporia (Polyporales, Basidiomycota) from China. MycoKeys 20, 37-50. Doi 10.3897/mycokeys.20.11816

Zhao CL, Cui BK. 2012 - A new species of Perenniporia (Polyporales, Basidiomycota) described from southern China based on morphological and molecular characters. Mycological Progress 11, 555-560. Doi 10.1007/s11557-011-0770-1

Zhou JL, Zhu L, Chen H, Cui BK. 2016 - Taxonomy and phylogeny of polyporus group Melanopus (Polyporales, Basidiomycota) from China. Plos One 11, e0159495. Doi 10.1371/journal.pone.0159495

Zhou JL, Cui BK. 2017 - Phylogeny and taxonomy of Favolus (Basidiomycota). Mycologia 109, 766-779. Doi 10.1080/00275514.2017.1409023

Zhou XS, Dai YC. 2008 - A new species of Megasporoporia (Polyporales, Basidiomycota) from China. Mycological Progress 7, 253-255. Doi 10.1007/s11557-008-0567-z

Zhu L, Song J, Zhou JL, Si J, Cui BK. 2019 - Species diversity, phylogeny, divergence time and biogeography of the genus Sanghuangporus (Basidiomycota). Frontiers in Microbiology 10, 812. Doi 10.3389/fmicb.2019.00812 